\title{
Bifurcations of periodic orbits and uniform approximations
}

\author{
Henning Schomerus $\dagger$ and Martin Sieber $\ddagger$ \\ $\dagger$ Fachbereich Physik, Universität-Gesamthochschule Essen, D-45117 Essen, Germany \\ $\ddagger$ Abteilung Theoretische Physik, Universität Ulm, D-89069 Ulm, Germany \\ Received 27 January 1997, in final form 2 May 1997
}

\begin{abstract}
We derive uniform approximations for contributions to Gutzwiller's periodic-orbit sum for the spectral density which are valid close to bifurcations of periodic orbits in systems with mixed phase space. There, orbits lie close together and give collective contributions, while the individual contributions of Gutzwiller's type would diverge at the bifurcation. New results for the tangent, the period-doubling and the period-tripling bifurcation are given. They are obtained by going beyond the local approximation and including higher-order terms in the normal form of the action. The uniform approximations obtained are tested on the kicked top and are found to be in excellent agreement with exact quantum results.
\end{abstract}

\section{Introduction}

Semiclassical approximations, in terms of periodic orbits, belong to the main tools for the examination of spectral properties of quantum systems. They allow us, for example, to explain fluctuations in quantum spectra in terms of the periodic orbits of the corresponding classical system. Semiclassical periodic-orbit approximations have been derived in cases where the classical dynamics is chaotic or integrable or has more general symmetries [1-8]. In these systems periodic orbits are typically either isolated or appear in families.

Most systems, however, are neither chaotic nor integrable but show a complicated mixture of regular and chaotic behaviour. In these systems, semiclassical approximations are more complicated and to date there do not exist complete semiclassical approximations for quantities such as the spectral density in terms of periodic orbits. These difficulties are due to the fact that in many situations periodic orbits neither appear in families nor can they be treated as being isolated in a semiclassical approximation. This is the case when there are other periodic orbits very near. If the action differences of neighbouring periodic orbits are not large in comparison with $\hbar$ then the orbits yield a collective semiclassical contribution, and this is the typical situation when bifurcations of periodic orbits occur. In order to extend semiclassical approximations to systems with mixed phase space one has to derive expressions for the joint contribution of orbits which participate in a bifurcation. For generic two-dimensional systems this was first done by de Almeida and Hannay [9]. They derived an approximation which was valid in the vicinity of a bifurcation. In this paper we extend the results of de Almeida and Hannay and derive analytic formulae which interpolate over the regime from a bifurcation up to regions where the orbits can be considered isolated.

Bifurcations are a characteristic phenomenon in systems with mixed phase space. They are responsible for the rapid increase of the number of periodic orbits when an integrable system is transformed into a chaotic system, for example by changing an external parameter. If one changes this parameter by an arbitrarily small but finite amount, then in general an 
infinite number of bifurcations occur, since they take place any time that the stability angle of a stable orbit is a rational multiple of $2 \pi$. There are different kinds of generic bifurcations, but the number of different forms is limited. The generic bifurcations that occur in twodimensional conservative systems (or, equivalently, one-dimensional area-preserving maps) were classified by Meyer and Bruno [10-12]. They are characterized by normal forms which describe the characteristic classical motion in the vicinity of a periodic orbit. Altogether one has to distinguish five different cases. These are the period- $m$-tupling bifurcations with $m=1$ up to $m=5$. They have the property that a central periodic orbit bifurcates and other periodic orbits split from the central orbit whose primitive period is $m$ times the primitive period of the central orbit. (An exception is the case $m=1$ for which there is no periodic orbit before the bifurcation.) The cases for $m>5$ follow the same pattern as for $m=5$.

By integrating over the normal forms de Almeida and Hannay derived their approximation. Their results are expressed in terms of diffraction catastrophe integrals. In the following we call this approximation the 'local approximation', since it is valid in the vicinity of a bifurcation. In farther distance from a bifurcation it reduces to an approximation of Gutzwiller's type for isolated periodic orbits, it does not yield, however, the correct semiclassical amplitudes of the orbits. In [13] the results of de Almeida were extended for the case $m \geqslant 5$ by including higher-order terms in the normal forms. A slightly generalized version of this result is given in [14]. In this paper we derive corrections for the low-order bifurcations with $m=1-3$. We obtain uniform approximations in closed form which are valid from a bifurcation up to the regime where Gutzwiller's approximation can be applied.

This paper is organized as follows. In section 2 we introduce the semiclassical method for treating bifurcations and in section 3 we present the results for the uniform approximations in two-dimensional conservative systems that are derived in the appendices. In section 4 we give the corresponding results for one-dimensional area-preserving maps. Numerical examinations of the uniform approximations are carried out in section 5 on the example of a kicked top, and in section 6 we discuss the range of validity of our approximations and possible extensions.

\section{Contributions to the spectral density}

In autonomous systems with discrete energy spectra the density of states

$$
d(E)=\sum_{n} \delta\left(E-E_{n}\right)=-\frac{1}{\pi} \operatorname{Im} \operatorname{Tr} G(E)
$$

can be expressed in terms of the trace of the (retarded) Green's function.

Semiclassical contributions of periodic orbits to the level density are derived from (1) by inserting the semiclassical approximation for the Green's function. In a mixed coordinatemomentum representation this approximation is given for two-dimensional systems by

$$
G\left(\boldsymbol{q}^{\prime}, \boldsymbol{p}, E\right) \approx \frac{1}{\mathrm{i} \hbar \sqrt{2 \pi \mathrm{i} \hbar}} \sum_{\xi} \sqrt{\left|D_{\xi}\right|} \exp \left\{\frac{\mathrm{i}}{\hbar} \hat{S}_{\xi}\left(\boldsymbol{q}^{\prime}, \boldsymbol{p}, E\right)-\frac{\mathrm{i} \pi}{2} \hat{\nu}_{\xi}\right\}
$$

where the sum runs over all classical trajectories with initial momentum $p$ and final position $\boldsymbol{q}^{\prime}$ at energy $E$. The function $\hat{S}_{\xi}$ for a trajectory $\xi$ is defined as

$$
\hat{S}_{\xi}\left(\boldsymbol{q}^{\prime}, \boldsymbol{p}, E\right)=\int_{\boldsymbol{q}}^{\boldsymbol{q}^{\prime}} \boldsymbol{p} \cdot \mathrm{d} \boldsymbol{q}+\boldsymbol{q} \cdot \boldsymbol{p}
$$


where the integral is evaluated along the trajectory $\xi$, and $q$ is the initial position of the trajectory which is determined by the initial momentum $\boldsymbol{p}$ and the final position $\boldsymbol{q}^{\prime}$. Its value follows from the condition that the gradient of the right-hand side of (3) with respect to $\boldsymbol{q}$ vanishes. $\hat{S}\left(\boldsymbol{q}^{\prime}, \boldsymbol{p}, E\right)$ is the generating function for a canonical transformation from final to initial coordinates of a trajectory. The mixed representation has been chosen since for a bifurcating orbit this transformation is close to the identity which cannot be generated in a pure coordinate or momentum representation.

Finally, $D_{\xi}$ is the determinant of a matrix of second derivatives of $\hat{S}_{\xi}$

$$
D_{\xi}=\operatorname{det}\left(\begin{array}{cc}
\frac{\partial^{2} \hat{S}_{\xi}}{\partial \boldsymbol{q}^{\prime} \partial \boldsymbol{p}} & \frac{\partial^{2} \hat{S}_{\xi}}{\partial \boldsymbol{q}^{\prime} \partial E} \\
\frac{\partial^{2} \hat{S}_{\xi}}{\partial E \partial p} & \frac{\partial^{2} \hat{S}_{\xi}}{\partial E^{2}}
\end{array}\right)
$$

and $\hat{v}_{\xi}$ is an integer related to the Maslov index (see, e.g. [7]).

The semiclassical spectral density is then determined by

$$
d(E)=-\frac{1}{\pi} \operatorname{Im} \int \mathrm{d}^{2} q^{\prime} \mathrm{d}^{2} p G\left(\boldsymbol{q}^{\prime}, \boldsymbol{p}, E\right) \exp \left(-\frac{\mathrm{i}}{\hbar} \boldsymbol{q}^{\prime} \cdot \boldsymbol{p}\right) .
$$

In the vicinity of a periodic orbit the integrals are evaluated by choosing local coordinates with one coordinate along the orbit and one perpendicular to it. If the integral over the perpendicular direction is evaluated in stationary phase approximation one obtains the Gutzwiller approximation for the semiclassical contribution of the orbit

$$
d_{\xi}(E)=\frac{1}{\pi \hbar} \frac{T_{\xi}}{r_{\xi} \sqrt{\left|\operatorname{Tr} M_{\xi}-2\right|}} \cos \left(\frac{S_{\xi}}{\hbar}-\frac{\pi}{2} v_{\xi}\right) .
$$

Here $S_{\xi}$ is the action of the orbit whose value is given by the function $\hat{S}_{\xi}\left(\boldsymbol{q}^{\prime}, \boldsymbol{p}, E\right)-\boldsymbol{q}^{\prime} \cdot \boldsymbol{p}$ for the considered periodic orbit. $T_{\xi}, M_{\xi}$ and $\nu_{\xi}$ are the period, stability matrix and Maslov index of the orbit, respectively. The integer $r_{\xi}$ denotes the repetition number of the orbit. In this notation we consider a periodic orbit and its multiple traversals which all give semiclassical contributions to the level density as different periodic orbits. The uniform approximations for bifurcating periodic orbits that are derived in this paper will be expressed in terms of exactly the same classical quantities that appear in (6).

We now consider the contribution of a bifurcating orbit with repetition number $r$ to the level density. The condition for such a bifurcation is that the stability matrix of the corresponding primitive periodic orbit (repetition number $r=1$ ) satisfies $\operatorname{Tr} M=$ $2 \cos (2 \pi n / r)$ with integers $r$ and $n$ and thus the stability matrix of the $r$ th traversal has a trace which is equal to two. Let $l$ be the greatest common divisor of $r$ and $n$. Then the bifurcation is a period- $m$-tupling bifurcation with $m=r / l$. Near the bifurcation the integral over the perpendicular coordinates cannot be evaluated in stationary phase approximation since there are stationary points nearby which correspond to the other orbits participating in the bifurcation. This is reflected by the fact that the Gutzwiller approximation (6) diverges at the bifurcation. Instead, one has to derive a joint contribution of all orbits which are involved in the bifurcation. This is achieved by expanding the generating function $\hat{S}\left(\boldsymbol{q}^{\prime}, \boldsymbol{p}, E\right)$ in higher order around the central orbit. In general this results in a complicated exponent in the integrand of (5). The integrals can be considerably simplified by a canonical transformation of the coordinates and by using the fact that the form of equation (5) is semiclassically invariant under canonical transformations. This follows from the work of Miller [15] and is discussed by Littlejohn [16].

The most simple form that the generating function can take near the bifurcation is given by the normal form. This normal form contains information about the number and arrangement of the orbits which are involved in the bifurcation. The transformation to the 
normal form coordinates has the further advantage that the integral over the coordinates along the periodic orbit can be performed trivially. These steps are explained in detail in [13] and for that reason we give here only the resulting formula

$d_{\xi}(E) \approx \frac{1}{2 \pi^{2} \hbar^{2}} \operatorname{Re} \int_{-\infty}^{\infty} \mathrm{d} q^{\prime} \int_{-\infty}^{\infty} \mathrm{d} p \frac{1}{r} \frac{\partial \hat{S}}{\partial E}\left|\frac{\partial^{2} \hat{S}}{\partial p \partial q^{\prime}}\right|^{\frac{1}{2}} \exp \left\{\frac{\mathrm{i}}{\hbar} \hat{S}\left(q^{\prime}, p, E\right)-\frac{\mathrm{i}}{\hbar} q^{\prime} p-\frac{\mathrm{i} \pi}{2} \nu\right\}$.

Here $p$ and $q^{\prime}$ are coordinates in the Poincaré surface of section perpendicular to the orbit, and $\hat{S}\left(q^{\prime}, p, E\right)$ is the generating function for the $r$ th iterate of the Poincare map which obeys the conditions

$$
\frac{\partial \hat{S}}{\partial q^{\prime}}=p^{\prime} \quad \frac{\partial \hat{S}}{\partial p}=q \quad \frac{\partial \hat{S}}{\partial E}=T
$$

where $T$ is the time from initial to final point.

The approximation of de Almeida and Hannay for the contributions of orbits near a bifurcation is obtained by inserting the normal form of the generating function $\hat{S}\left(q^{\prime}, p, E\right)$ for a particular generic bifurcation. This yields the semiclassical contributions in terms of standard diffraction catastrophe integrals. For the bifurcations which are considered in this paper the normal forms are given by

$$
\begin{array}{ll}
m=1: & \hat{S}\left(q^{\prime}, p, E\right)=S_{0}(E)+q^{\prime} p-\frac{\sigma}{2} p^{2}-\varepsilon q^{\prime}-a q^{\prime 3} \\
m=2: & \hat{S}\left(q^{\prime}, p, E\right)=S_{0}(E)+q^{\prime} p-\frac{\sigma}{2} p^{2}-\varepsilon q^{\prime 2}-a q^{\prime 4} \\
m=3: & \hat{S}\left(q^{\prime}, p, E\right)=S_{0}(E)+q^{\prime} p-\frac{\varepsilon}{2}\left(q^{\prime 2}+p^{2}\right)-\frac{a}{\sqrt{8}}\left(p^{3}-3 p q^{\prime 2}\right)
\end{array}
$$

where $\varepsilon$ is a parameter which is zero at the bifurcation and $\sigma$ is a sign factor. As will be shown in the appendices, the diffraction integrals for these cases can be expressed in terms of Bessel functions. The properties of the bifurcations corresponding to these normal forms are discussed in more detail in the next section. All these bifurcations involve two periodic orbits.

The approximation of de Almeida and Hannay is valid in the vicinity of a bifurcation and hence we called it the local approximation. Further from a bifurcation the local approximation splits up asymptotically into a sum of separate contributions of Gutzwiller's type for the periodic orbits. However, in this limit the semiclassical amplitudes of the orbits have a fixed ratio, i.e. there is a fixed relationship between the stabilities (and periods) of the different orbits. In more detail, the approximation holds when the following relations between the monodromy matrices and the periods of the orbits are valid.

$$
\begin{array}{lll}
m=1: & \operatorname{Tr} M_{1}+\operatorname{Tr} M_{2}-4=0 & T_{1}=T_{2} \\
m=2: & \operatorname{Tr} M_{1}+2 \operatorname{Tr} M_{0}-6=0 & T_{1}=T_{0} \\
m=3: & \operatorname{Tr} M_{1}+3 \operatorname{Tr} M_{0}-8=0 & T_{1}=T_{0} .
\end{array}
$$

For $m=2$ and $m=3$ the index 0 denotes the central bifurcating orbit and the index 1 denotes the satellite orbit. For the case $m=1$ there is no central periodic orbit and the two periodic orbits which are involved in the bifurcation are given the indices 1 and 2. Relations (10) follow from the normal forms and are valid in the vicinity of a bifurcation. Further from a bifurcation, however, they are not valid any more, and then the local approximation becomes inaccurate. In order to obtain a formula which uniformly interpolates over the region from the bifurcation up to regimes where the Gutzwiller 
approximation is valid (without restrictions on the semiclassical amplitudes) one has to include higher-order corrections to the normal forms in (9). The resulting integrals can then be reduced to simpler forms by appropriate coordinate transformations. These calculations are carried out in appendices $\mathrm{A}-\mathrm{C}$, and the results are discussed in the next section.

\section{Results for the uniform approximations}

\subsection{The isochronuous bifurcation}

The bifurcation which is described by the normal form in (9) for $m=1$ has the following property: on one side of the bifurcation where $\varepsilon$ and $a$ have opposite signs two periodic orbits exist, one stable and one unstable. We denote these orbits by $\xi_{1}$ and $\xi_{2}$. On the other side of the bifurcation where $\varepsilon$ and $a$ have the same sign both orbits are complex, and we give the index 1 to that orbit for which the imaginary part of the action is positive. Due to the shape of the function $\hat{S}\left(q^{\prime}, p, E\right)-q^{\prime} p$ this bifurcation is called tangent bifurcation or saddle-node bifurcation.

The uniform approximation for the semiclassical contributions of the two orbits is derived in appendix A. Its form is different on the two sides of the bifurcation. On the side where the orbits are real it is given by

$$
\begin{aligned}
d_{\xi}(E)=\frac{1}{\pi \hbar} \mid & \left.\frac{2 \pi \Delta S}{3 \hbar}\right|^{1 / 2}\left\{\frac{A_{1}+A_{2}}{2} \cos \left(\frac{\bar{S}}{\hbar}-\frac{\pi}{2} \bar{v}\right)\left(J_{-1 / 3}\left(\frac{|\Delta S|}{\hbar}\right)+J_{1 / 3}\left(\frac{|\Delta S|}{\hbar}\right)\right)\right. \\
& -\operatorname{sign}(\Delta S) \frac{A_{1}-A_{2}}{2} \cos \left(\frac{\bar{S}}{\hbar}-\frac{\pi}{2}(\bar{v}-1)\right) \\
& \left.\times\left(J_{-2 / 3}\left(\frac{|\Delta S|}{\hbar}\right)-J_{2 / 3}\left(\frac{|\Delta S|}{\hbar}\right)\right)\right\}
\end{aligned}
$$

which is invariant under exchange of the two indices. The quantities which appear in (11) are the mean action $\bar{S}=\left(S_{1}+S_{2}\right) / 2$, the action difference $\Delta S=\left(S_{1}-S_{2}\right) / 2$ and the mean Maslov index $\bar{v}=\left(v_{1}+v_{2}\right) / 2=v+\sigma / 2$ of the orbits. Here $v$ is the index in (7) and $\sigma$ is the sign factor in the normal form (9). Furthermore, $A_{i}$ denotes here and in the following the semiclassical amplitude of an orbit

$$
A_{i}=\frac{T_{i}}{r_{i} \sqrt{\eta\left(\operatorname{Tr} M_{i}-2\right)}}
$$

where $\eta$ is given by $\eta=\operatorname{sign}\left(\operatorname{Re}\left(\operatorname{Tr} M_{i}-2\right)\right)$ except for $m=1$ in case that both orbits are complex. Then $\eta=-\mathrm{i} \operatorname{sign}\left(\operatorname{Im}\left(\operatorname{Tr} M_{i}\right)\right)$.

Properties of the two orbits when they are real are listed in table 1, and an expansion of the classical properties of the orbits in terms of the coefficients in the (extended) normal form are given in appendix A.

Table 1. Properties of real orbits that participate in a generic isochronuous bifurcation $(m=1)$. The orbits are denoted by $\xi_{u}$ and $\xi_{s}$ where the indices $u$ and $s$ denote the unstable and stable orbit, respectively.

\begin{tabular}{lll}
\hline$m=1$ & $\sigma>0$ & $\sigma<0$ \\
\hline$a \varepsilon<0$ & $\xi_{u}$ unstable, $v_{u}=v$ & $\xi_{u}$ unstable, $v_{u}=v$ \\
& $\xi_{s}$ stable, $v_{s}=v+1$ & $\xi_{s}$ stable, $v_{s}=v-1$ \\
& $S_{s}>S_{u}$ & $S_{u}>S_{s}$ \\
\hline
\end{tabular}


On the other side of the bifurcation where the orbits are complex the semiclassical contribution to the level density is given by

$$
\begin{aligned}
d_{\xi}(E)=\operatorname{Re}[ & \frac{1}{\pi \hbar}\left|\frac{2 \Delta S}{\pi \hbar}\right|^{1 / 2} \exp \left(\frac{\mathrm{i}}{\hbar} \bar{S}-\frac{\mathrm{i} \pi}{2} v-\frac{\mathrm{i} \pi}{4} \sigma\right) \\
& \left.\times\left\{\frac{A_{1}+A_{2}}{2} K_{1 / 3}\left(\frac{|\Delta S|}{\hbar}\right)+\frac{A_{1}-A_{2}}{2} K_{2 / 3}\left(\frac{|\Delta S|}{\hbar}\right)\right\}\right]
\end{aligned}
$$

where now the actions and amplitudes are complex. Again $v$ is the index in (7) and $\sigma$ is the sign factor in the normal form (9). Equations (11) and (13) can be written in a combined form by expressing them in terms of an Airy function.

In the limit $\varepsilon \rightarrow 0$ the leading-order semiclassical contribution of equations (11) and (13) is given by

$$
d_{\xi}(E)=\frac{T_{0} \Gamma \frac{1}{3}}{\pi l \sqrt{6 \pi} \hbar^{7 / 6} a^{1 / 3}} \cos \left(\frac{S_{0}}{\hbar}-\frac{\pi}{2} \nu-\frac{\pi}{2} \sigma\right) .
$$

Here $T_{0}$ and $S_{0}$ are, respectively, the period and action of the orbits at the bifurcation, $a$ is the coefficient in the normal form (9), and $l$ is the repetition number of the bifurcating orbits. The contribution (14) is of order $\hbar^{-1 / 6}$ larger than the contribution of an isolated period orbit. All appearing classical quantities in (14) depend on the integer $l$. In detail, $T_{0, l}=l T_{0, l=1}$, $S_{0, l}=l S_{0, l=1}, v_{l}=l v_{l=1}$ and $a_{l}=l^{5 / 2} a_{l=1}$. The relation for the Maslov index follows from the Maslov index of the unstable orbit in table 1 for multiple traversals. The last relation is obtained by noting that all coefficients in the normal form (9) increase by a factor of $l$ as $l$ is increased. However, $\sigma$ is restricted to be \pm 1 . This is achieved by a subsequent (canonical) transformation $p \rightarrow p / \sqrt{l}$ and $q \rightarrow q \sqrt{l}$. It follows that the amplitude of the contribution at the bifurcation decreases like $l^{-5 / 6}$ with $l$. However, this result is only valid as long as the bifurcation can be considered isolated from other bifurcations. Longer periodic orbits tend to bifurcate more and more rapidly, for that reason contribution (14) is expected to break down for large $l$ since the bifurcation cannot then be considered isolated from further bifurcations of the participating orbits.

\subsection{The period-doubling bifurcation}

The period-doubling bifurcation which is described by the normal form in (9) for $m=2$ has the following form. On one side of the bifurcation where $\varepsilon$ and $a$ have the same sign there is only one orbit which is called the central orbit. This orbit changes its stability at the bifurcation from stable to unstable or vice versa, and a new orbit appears which is named the satellite orbit since the two fixed points of the Poincaré map which belong to this orbit lie symmetrically on both sides of the fixed point of the central orbit. This bifurcation is also called pitchfork bifurcation.

The uniform approximation for the contributions of these orbits to the level density is derived in appendix $\mathrm{B}$ and is given by

$$
\begin{aligned}
d_{\xi}(E)=\operatorname{Re}\left[\frac{1}{\pi \hbar}\left|\frac{\pi \Delta S}{2 \hbar}\right|^{1 / 2} \exp \left(\frac{\mathrm{i}}{\hbar} \bar{S}-\frac{\mathrm{i} \pi}{2} v-\frac{i \pi}{4} \sigma\right)\right. \\
\times\left\{\left(\frac{A_{1}}{2}+\frac{A_{0}}{\sqrt{2}}\right)\left(\sigma_{2} J_{1 / 4}\left(\frac{|\Delta S|}{\hbar}\right) \mathrm{e}^{\mathrm{i} \sigma_{1} \pi / 8}+J_{-1 / 4}\left(\frac{|\Delta S|}{\hbar}\right) \mathrm{e}^{-\mathrm{i} \sigma_{1} \pi / 8}\right)\right. \\
\left.\left.+\left(\frac{A_{1}}{2}-\frac{A_{0}}{\sqrt{2}}\right)\left(J_{3 / 4}\left(\frac{|\Delta S|}{\hbar}\right) \mathrm{e}^{\mathrm{i} \sigma_{1} 3 \pi / 8}+\sigma_{2} J_{-3 / 4}\left(\frac{|\Delta S|}{\hbar}\right) \mathrm{e}^{-\mathrm{i} \sigma_{1} 3 \pi / 8}\right)\right\}\right] .
\end{aligned}
$$


Table 2. Properties of real orbits that participate in a generic period-doubling bifurcation $(m=2)$. The central orbit is denoted by $\xi_{0}$ and the satellite orbit is denoted by $\xi_{1}$.

\begin{tabular}{|c|c|c|c|c|}
\hline$m=2$ & $\sigma>0$ & & $\sigma<0$ & \\
\hline$a>0$ & $\begin{array}{l}\varepsilon>0: \\
\varepsilon<0:\end{array}$ & $\begin{array}{l}\xi_{0} \text { stable, } v_{0}=v+1 \\
\xi_{0} \text { unstable, } v_{0}=v \\
\xi_{1} \text { stable, } v_{1}=v+1 \\
S_{1}>S_{0}\end{array}$ & $\begin{array}{l}\varepsilon>0: \\
\varepsilon<0:\end{array}$ & $\begin{array}{l}\xi_{0} \text { unstable, } v_{0}=v \\
\xi_{0} \text { stable, } v_{0}=v-1 \\
\xi_{1} \text { unstable, } v_{1}=v \\
S_{1}>S_{0}\end{array}$ \\
\hline$a<0$ & $\begin{array}{l}\varepsilon<0: \\
\varepsilon>0:\end{array}$ & $\begin{array}{l}\xi_{0} \text { unstable, } v_{0}=v \\
\xi_{0} \text { stable, } v_{0}=v+1 \\
\xi_{1} \text { unstable, } v_{1}=v \\
S_{1}<S_{0}\end{array}$ & $\begin{array}{l}\varepsilon<0: \\
\varepsilon>0:\end{array}$ & $\begin{array}{ll}\xi_{0} & \text { stable, } v_{0}=v-1 \\
\xi_{0} & \text { unstable, } v_{0}=v \\
\xi_{1} & \text { stable, } v_{1}=v-1 \\
S_{1}<S_{0}\end{array}$ \\
\hline
\end{tabular}

This approximation is valid on both sides of the bifurcation. Here $\bar{S}=\left(S_{1}+S_{0}\right) / 2$, $\Delta S=\left(S_{1}-S_{0}\right) / 2, v$ is the index in (7) and $\sigma$ is the sign factor in the normal form (9). The values of $v$ and $\sigma$ can be determined from the properties of the orbits which are listed in table 2. Furthermore, $\sigma_{1}=\operatorname{sign}(\Delta S)$ and $\sigma_{2}$ is a sign factor which discriminates between both sides of the bifurcation. It is 1 when both orbits are real and -1 when only the central orbit is real. The orbit $\xi_{1}$ also contributes to (15) when it is complex, but its action and amplitude factor are always real. The expression of the classical properties of the two orbits in terms of the coefficients in the (extended) normal form are given in appendix B.

In the limit $\varepsilon \rightarrow 0$ the leading-order semiclassical contribution of equation (15) is given by

$$
d_{\xi}(E)=\frac{T_{0} \Gamma^{\frac{1}{4}}}{4 \pi l \sqrt{2 \pi} \hbar^{5 / 4} a^{1 / 4}} \cos \left(\frac{S_{0}}{\hbar}-\frac{\pi}{2} \nu-\frac{\pi}{4} \sigma-\frac{\pi}{8} \sigma_{1}\right) .
$$

Here $T_{0}$ and $S_{0}$ are, respectively, the period and action of the orbits at the bifurcation, $a$ is the coefficient in the normal form (9), and $l$ is the repetition number of the orbit $\xi_{1}$. The contribution (16) is of order $\hbar^{-1 / 4}$ larger than the contribution of an isolated period orbit. The dependence of the classical quantities in (16) on the integer $l$ are given by: $T_{0, l}=l T_{0, l=1}, S_{0, l}=l S_{0, l=1}, v_{l}=l v_{l=1}$ and $a_{l}=l^{3} a_{l=1}$. The relation for the Maslov index follows from the Maslov index of the unstable orbit in table 2 for multiple traversals, and the relation for the coefficient $a$ from considerations analogous to those for the isochronuous bifurcation. It follows that the amplitude of the contribution at the bifurcation decreases like $l^{-3 / 4}$ with $l$.

\subsection{The period-tripling bifurcation}

The period-tripling bifurcation is described by the normal form in (9) for $m=3$. It involves two orbits, the central orbit $\xi_{0}$ and the satellite orbit $\xi_{1}$. Both orbits exist before and after the bifurcation. As $\varepsilon$ goes through zero both orbits approach each other, they coincide at the bifurcation and then separate again. For that reason this bifurcation has also been named 'touch and go' bifurcation [17].

The uniform approximation for this bifurcation is derived in appendix $\mathrm{C}$ and is given 
Table 3. Properties of orbits that participate in a generic period-tripling bifurcation $(m=3)$. The central orbit is denoted by $\xi_{0}$ and the satellite orbit by $\xi_{1}$.

\begin{tabular}{lll}
\hline$m=3$ & $\varepsilon>0$ & $\varepsilon<0$ \\
\hline$\xi_{0}$ stable, $v_{0}=v+1$ & $\xi_{0}$ stable, $v_{0}=v-1$ \\
& $\xi_{1}$ unstable, $v_{1}=v$ & $\xi_{1}$ unstable, $v_{1}=v$ \\
& $S_{1}<S_{0}$ & $S_{1}>S_{0}$ \\
\hline
\end{tabular}

by

$$
\begin{aligned}
d_{\xi}(E)=\frac{1}{\pi \hbar} & \operatorname{Re} \sqrt{\frac{2 \pi|\Delta S|}{\hbar}} \exp \left\{\frac{\mathrm{i}}{\hbar} \bar{S}-\frac{\mathrm{i} \pi}{2} \nu\right\} \\
& \times\left\{\left(\frac{A_{0}}{2}+\frac{A_{1}}{2 \sqrt{3}}\right)\left[J_{-1 / 6}\left(\frac{|\Delta S|}{\hbar}\right)+\mathrm{i} \sigma J_{1 / 6}\left(\frac{|\Delta S|}{\hbar}\right)\right]\right. \\
& \left.-\left(\frac{A_{0}}{2}-\frac{A_{1}}{2 \sqrt{3}}\right)\left[J_{-5 / 6}\left(\frac{|\Delta S|}{\hbar}\right)+\mathrm{i} \sigma J_{5 / 6}\left(\frac{|\Delta S|}{\hbar}\right)\right]\right\} .
\end{aligned}
$$

Here $\bar{S}=\left(S_{1}+S_{0}\right) / 2, \Delta S=\left(S_{1}-S_{0}\right) / 2, v=v_{1}$ is the Maslov index of the satellite orbit. Furthermore $\sigma=\operatorname{sign}(\Delta S)$. Properties of the two orbits are listed in table 3 , and the expression of the classical properties of the two orbits in terms of the coefficients in the (extended) normal form are given in appendix $\mathrm{C}$. by

In the limit $\varepsilon \rightarrow 0$ the leading-order semiclassical contribution of equation (17) is given

$$
d_{\xi}(E)=\frac{T_{0} \Gamma \frac{1}{6}}{9 l \pi^{3 / 2} \hbar^{4 / 3} a^{2 / 3}} \cos \left(\frac{S_{0}}{\hbar}-\frac{\pi}{2} v\right) .
$$

Here $T_{0}$ and $S_{0}$ are, respectively, the period and action of the orbits at the bifurcation, $a$ is the coefficient in the normal form (9), and $l$ is the repetition number of the orbit $\xi_{1}$. Contribution (18) is of order $\hbar^{-1 / 3}$ larger than the contribution of an isolated period orbit. The dependence of the classical quantities in (18) on the integer $l$ are given by: $T_{0, l}=l T_{0, l=1}, S_{0, l}=l S_{0, l=1}, v_{l}=l v_{l=1}$ and $a_{l}=l a_{l=1}$. The relation for the Maslov index follows from the Maslov index of the unstable orbit in table 3 for multiple traversals. It follows that the amplitude of the contribution at the bifurcation decreases like $l^{-2 / 3}$ with $l$.

\section{Uniform approximations for maps}

Although we concentrated on autonomous systems in the preceding paragraphs, the uniform contributions given there can equally be applied, with minor modifications, to quantum maps. These maps are described by a unitary time-evolution operator $F$, the so-called Floquet operator; the dynamics of the system is generated by repeated applications of the operator on a state in Hilbert space. $F$ has eigenstates and unimodular eigenvalues $\mathrm{e}^{-\mathrm{i} \varphi_{i}}$, where the phases $\varphi_{i}$ are called quasi-energies and the states quasistationary states since many quantum maps originate from a stroboscopic description of periodically driven systems. For such systems with Hamiltonian $H(t+T)=H(t)$, the unitary time-evolution operator after period $T$ is chosen as $F=U(T)$, implying $U(n T)=F^{n}$.

Restricting oneself onto a finite-dimensional subspace of Hilbert space with dimension 
$N$ the quasi-energy spectrum can be obtained by solving the secular equation

$$
P\left(\lambda=\mathrm{e}^{-\mathrm{i} \varphi}\right):=\operatorname{det}(F-\lambda I)=\sum_{n=0}^{N} a_{n} \lambda^{N-n}=0
$$

for the $N \times N$-matrix $F$. Here the set of traces $\operatorname{Tr} F^{n}$ comes into play: their knowledge for $n$ up to $N / 2$ allows us to construct the first half of the coefficients $a_{n}$ via the so-called Newton formulae; the other half follows from the unitarity of $F$ which entails self-inversiveness of the secular polynomial [18].

The semiclassical starting point for the calculation of $\operatorname{Tr} F^{n}$ is an expression which is nearly identical in its appearance with equation (7) for autonomous systems,

$$
\operatorname{Tr} F^{n}=\iint \frac{\mathrm{d} p \mathrm{~d} q}{2 \pi \hbar}\left|\frac{\partial^{2} \hat{S}}{\partial p \partial q^{\prime}}\right|^{\frac{1}{2}} \exp \left\{\frac{\mathrm{i}}{\hbar}\left(\hat{S}\left(q^{\prime}, p ; n T\right)-q^{\prime} p\right)-\mathrm{i} \frac{\pi}{2} \nu\right\} .
$$

The major difference to the expression for autonomous systems is that one does not switch to energy representation by a Fourier transform with respect to time $t$. In translating the contributions which were derived for autonomous systems into the appropriate contributions to $\operatorname{Tr} F^{n}$ attention has to be paid to the following five differences. (i) The orbits which contribute are those with a fixed period $n$, not those with a given energy $E$; (ii) the primitive periods have to be expressed in units of $T$ and thus are integer valued; (iii) the action is not the reduced energy-dependent one, but depends on time (that is, on the number $n$ ); (iv) instead of taking twice the real part, the full complex contribution has to be taken; (v) the results further differ by a factor of $2 \pi \hbar$. Since these are mainly formal differences the morphology of the contributions remains unaltered.

In the limit $\hbar \rightarrow 0$ each orbit of primitive period $n_{0}$ contributes individually according to

$$
\operatorname{Tr} F^{n}=\sum_{\text {orbits }} \frac{n_{0}}{|2-\operatorname{Tr} M|^{1 / 2}} \exp \left\{\frac{\mathrm{i}}{\hbar} S-\mathrm{i} \frac{\pi}{2} \nu\right\} .
$$

In the following we denote the contributions to $\operatorname{Tr} F^{n}$ by $C^{(n)}$ and use $A_{i}=n_{0, i}\left(\eta\left(\operatorname{Tr} M_{i}-\right.\right.$ 2) $)^{-1 / 2}$ to abbreviate the stability amplitudes. $\eta$ is given by $\eta=\operatorname{sign}\left(\operatorname{Re}\left(\operatorname{Tr} M_{i}-2\right)\right)$ except if $m=1$ and both orbits are complex, then $\eta=-\mathrm{i} \operatorname{sign}\left(\operatorname{Im}\left(\operatorname{Tr} M_{i}\right)\right) \cdot \bar{S}=\left(S_{1}+S_{i}\right) / 2$ is the mean action and $\Delta S=\left(S_{1}-S_{i}\right) / 2$ the action difference where $i$ is 0 or 2 depending on the considered bifurcation, and $v$ is the index in (19).

For $m=1$ the collective contribution is

$$
\begin{aligned}
C^{(n)}=\mid \frac{2 \pi \Delta S}{3 \hbar} & \left.\right|^{1 / 2}\left\{\frac{A_{1}+A_{2}}{2} \exp \left(\mathrm{i} \frac{\bar{S}}{\hbar}-\mathrm{i} \frac{\pi}{2} \bar{v}\right)\left(J_{-1 / 3}\left(\frac{|\Delta S|}{\hbar}\right)+J_{1 / 3}\left(\frac{|\Delta S|}{\hbar}\right)\right)\right. \\
& -\operatorname{sign}(\Delta S) \frac{A_{1}-A_{2}}{2} \exp \left(\mathrm{i} \frac{\bar{S}}{\hbar}-\mathrm{i} \frac{\pi}{2}(\bar{v}-1)\right) \\
& \left.\times\left(J_{-2 / 3}\left(\frac{|\Delta S|}{\hbar}\right)-J_{2 / 3}\left(\frac{|\Delta S|}{\hbar}\right)\right)\right\}
\end{aligned}
$$

when both orbits are real. When they are complex one obtains

$$
\begin{aligned}
C^{(n)}=\left|\frac{2 \Delta S}{\pi \hbar}\right|^{1 / 2} & \exp \left(\frac{\mathrm{i}}{\hbar} \bar{S}-\frac{\mathrm{i} \pi}{2} v-\frac{\mathrm{i} \pi}{4} \sigma\right) \\
& \times\left\{\frac{A_{1}+A_{2}}{2} K_{1 / 3}\left(\frac{|\Delta S|}{\hbar}\right)+\frac{A_{1}-A_{2}}{2} K_{2 / 3}\left(\frac{|\Delta S|}{\hbar}\right)\right\} .
\end{aligned}
$$


The amplitudes $A_{i}$ are now complex quantities. $\sigma$ is the sign factor in the normal form (9). A pair of orbits involved in a period doubling bifurcation $(m=2)$ gives the contribution

$$
\begin{aligned}
C^{(n)}=\left|\frac{\pi \Delta S}{2 \hbar}\right|^{1 / 2} & \exp \left(\frac{\mathrm{i}}{\hbar} \bar{S}-\frac{\mathrm{i} \pi}{2} v-\frac{\mathrm{i} \pi}{4} \sigma\right) \\
& \times\left\{\left(\frac{A_{1}}{2}+\frac{A_{0}}{\sqrt{2}}\right)\left(\sigma_{2} J_{1 / 4}\left(\frac{|\Delta S|}{\hbar}\right) \mathrm{e}^{\mathrm{i} \sigma_{1} \pi / 8}+J_{-1 / 4}\left(\frac{|\Delta S|}{\hbar}\right) \mathrm{e}^{-\mathrm{i} \sigma_{1} \pi / 8}\right)\right. \\
+ & \left.\left(\frac{A_{1}}{2}-\frac{A_{0}}{\sqrt{2}}\right)\left(J_{3 / 4}\left(\frac{|\Delta S|}{\hbar}\right) \mathrm{e}^{\mathrm{i} \sigma_{1} 3 \pi / 8}+\sigma_{2} J_{-3 / 4}\left(\frac{|\Delta S|}{\hbar}\right) \mathrm{e}^{-\mathrm{i} \sigma_{1} 3 \pi / 8}\right)\right\}
\end{aligned}
$$

with $\sigma_{1}=\operatorname{sign}(\Delta S)$ and $\sigma_{2}=1$ when the satellite is real, $\sigma_{2}=-1$ otherwise. For $m=3$ the result reads

$$
\begin{gathered}
C^{(n)}=\sqrt{\frac{2 \pi|\Delta S|}{\hbar}} \exp \left\{\frac{\mathrm{i}}{\hbar} \bar{S}-\frac{\mathrm{i} \pi}{2} v\right\}\left\{\left(\frac{A_{0}}{2}+\frac{A_{1}}{2 \sqrt{3}}\right)\left[J_{-1 / 6}\left(\frac{|\Delta S|}{\hbar}\right)+\mathrm{i} \sigma J_{1 / 6}\left(\frac{|\Delta S|}{\hbar}\right)\right]\right. \\
\left.-\left(\frac{A_{0}}{2}-\frac{A_{1}}{2 \sqrt{3}}\right)\left[J_{-5 / 6}\left(\frac{|\Delta S|}{\hbar}\right)+\mathrm{i} \sigma J_{5 / 6}\left(\frac{|\Delta S|}{\hbar}\right)\right]\right\}
\end{gathered}
$$

with $\sigma=\operatorname{sign}(\Delta S)$.

These expressions also give the contribution of repetitions of the bifurcating orbits if one substitutes the corresponding classical quantities. For the $l$ th repetition, the stability angle $\omega$ of a stable orbit in $\operatorname{Tr} M=2 \cos \omega$ and the instability exponent $u$ of an unstable orbit in $\operatorname{Tr} M= \pm 2 \cosh u$ increase linearly, $\omega_{l}=l \omega_{l=1}$ and $u_{l}=l u_{l=1}$, as does the action $S_{l}=l S_{l=1}$ and the Maslov index of the unstable orbits $v_{l}^{(u)}=l v_{l=1}^{(u)}$. The Maslov index of the stable orbits is $v_{l}^{(s)}=v_{l}^{(u)}+\operatorname{sign}\left(S_{l}^{(s)}-S_{l}^{(u)}\right)$.

\section{Numerical results}

In this section we want to test the quality of the uniform collective contributions that we derived for the various types of bifurcations on a certain quantum map, a periodically kicked top [19-21]. It will turn out that the uniform approximations are indeed accurate both close to the bifurcation as well as at a distance; the local approximation is only valid close to the bifurcation while far away the orbits can be treated as being isolated via the stationary-phase approximation.

Tops are dynamical systems that involve the angular-momentum operators $J_{x}, J_{y}, J_{z}$, satisfying the usual commutation relations $\left[J_{k}, J_{l}\right]=\mathrm{i} \epsilon_{k l m} J_{m}$, where $\hbar$ is set to unity. The evolution of the system is such that the total angular momentum $J_{x}^{2}+J_{y}^{2}+J_{z}^{2}=j(j+1)$ is conserved. This introduces the well known good quantum number $j$ which fixes the Hilbert space dimension $2 j+1$. $j+\frac{1}{2}$ further plays the role of the inverse of Planck's constant; accordingly, the semiclassical limit is reached by sending $j \rightarrow \infty$.

The specific top used here is described by the Floquet operator

$F=\exp \left(-\mathrm{i} \frac{k_{z}}{2 j+1} J_{z}^{2}-\mathrm{i} p_{z} J_{z}\right) \exp \left(-\mathrm{i} p_{y} J_{y}\right) \exp \left(-\mathrm{i} \frac{k_{x}}{2 j+1} J_{x}^{2}-\mathrm{i} p_{x} J_{x}\right)$.

The dynamics consists of rotations by angles $p_{i}$ and nonlinear rotations (torsions) of strength $k_{i}$. For the study of bifurcations we hold the $p_{i}$ at fixed values $\left(p_{x}=0.3, p_{y}=1.0\right.$, $p_{z}=0.8$ ) and take $k=k_{z}$ as our control parameter, with $k_{x}=k / 10$. The classical counterpart of the system is integrable at $k=0$ and displays well developed chaos at $k=5$. 
action spectrum
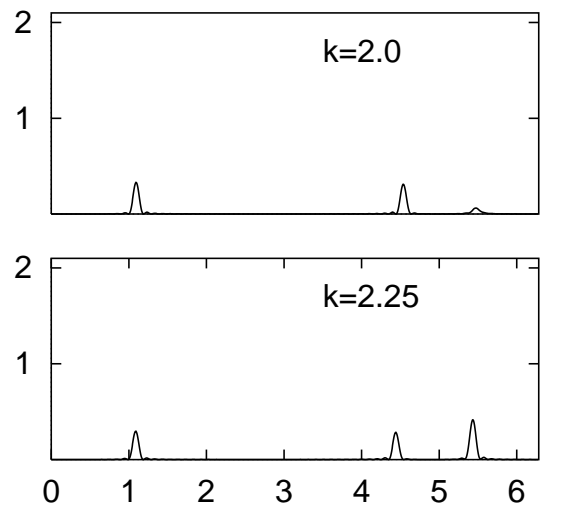

action spectrum
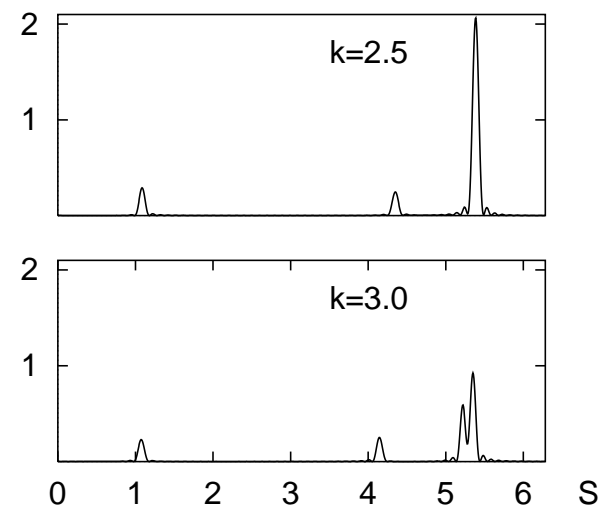

Figure 1. Sequence of action spectra $\left|T^{(1)}(S)\right|^{2}$ as the control parameter crosses a tangent bifurcation $(m=1)$ at $k=2.45$. A ghost peak shows up before the bifurcation at $S=5.3$; beyond the bifurcation the peak splits into two, each corresponding to a bifurcating orbit.

There is a very convenient testing tool which enables one to examine each contribution of a given cluster of periodic orbits individually. It is obtained by considering the function

$$
T^{(n)}(S)=\frac{1}{j_{\max }-j_{\min }+1} \sum_{j=j_{\min }}^{j_{\max }} \mathrm{e}^{-\mathrm{i} j S} \operatorname{Tr} F^{n}(j) .
$$

In its essence this is a Fourier coefficient of $\operatorname{Tr} F^{n}$ with respect to $j$; finite limits have to be taken for practical reasons if one wants to evaluate the sum for the quantum system, they further give control over the desired degree of rigor of the semiclassical limit. We used $j_{\min }=1$ and $j_{\max }=64$ with a single exception for $m=3$.

From the asymptotic behaviour (20) of all uniform approximations it is clear that one expects peaks in $\left|T^{(n)}(S)\right|^{2}$ at values of the argument which correspond to actions of periodic orbits. The function $\left|T^{(n)}(S)\right|^{2}$ is called the action spectrum for that reason. Figure 1 confirms that peaks indeed show up. A sequence of action spectra is plotted for $n=1$ as the control parameter, $k$ is varied across a tangent bifurcation at $k=2.45$. Already before the pair of orbits comes into existence a peak is visible at $S=5.3$. This peak arises from the complex predecessors of the bifurcating orbits that were already observed in [22]. The other peaks pertain to different orbits. Slightly beyond the bifurcation the new-born orbits have nearly identical actions and give rise to a single peak that would be resolved if one would go to much higher $j_{\max }$. Increasing the control parameter further the peak splits into two peaks which are located at the now well separated values of the orbits' actions.

The quality of our results can now be tested by calculating the height of the peaks $\left|T^{(n)}\left(S_{\mathrm{cl}}\right)\right|^{2}$ both quantum-mechanically exact as well as on the grounds of the various semiclassical approximations. Figure 2 depicts the height of the aforementioned peaks as the control parameter is varied across the tangent bifurcation. In the vicinity of the bifurcation both the local, as well as the uniform approximation, are accurate to a degree that makes it difficult to resolve the error at all. The uniform approximation also remains valid as one moves away from the bifurcation. There the sum of individual contributions of isolated orbits is seen to gain validity.

Figure 3 illustrates the superiority of the uniform approximation over the local one away from the bifurcation. To that end, the exact peak itself is compared with the approximated 


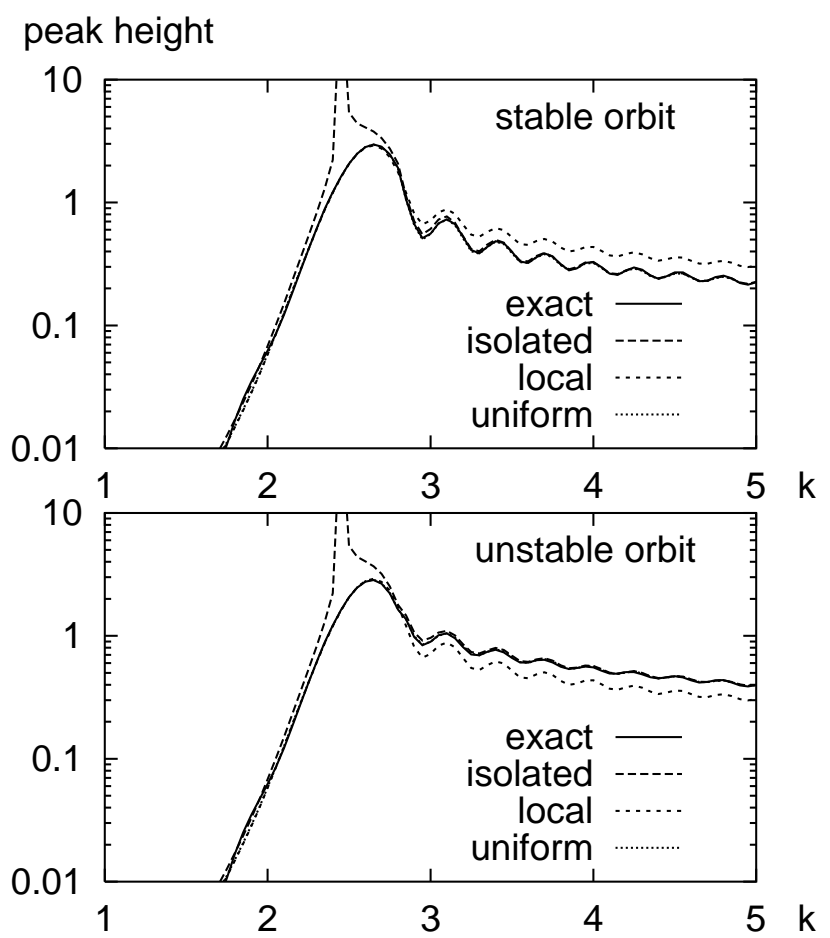

Figure 2. Height of the peaks in the action spectrum at the positions of the two orbits that are engaged in the bifurcation of figure 1. Both the uniform and the local bifurcations work well close to and on the left of the bifurcation. The local approximation starts to fail as $k$ is increased, and the sum of isolated contributions of Gutzwiller's type becomes valid. Note that the uniform approximation remains accurate and can hardly be distinguished from the exact result in this plot.

ones. The local approximation assumes that the stabilities of both orbits are equal and gives two peaks of the same height. With the sum of two isolated terms of Gutzwiller's type both peak heights are slightly overestimated. The uniform approximation almost coincides with the exact result. Figure 3 thus shows a case where the distance from the bifurcation is already so large that the local approximation becomes bad, but not large enough for the Gutzwiller approximation to become as good as the uniform approximation.

In figure 4, the peak heights of orbits participating in a period doubling at $k=4.3$ are plotted. In this case it is hardly possible to discriminate between the local and the uniform approximations. Before the amplitudes start to differ significantly, erratic deviations to the exact result are encountered which stem from overlapping peaks of other orbits. One such orbit has already been included here with its isolated contribution for $k>5.8$ and cures part of the problem, but other orbits become relevant from $k \approx 6.2$. Instead of further improving on this we settle for the information that the approximations work well close to the bifurcation and turn to other period doublings. Figure 5 actually arises from a similar situation of two overlapping peaks; both peaks, however, arise from two pairs of orbits which undergo period doublings at $k=2.78$ and $k=2.84$, respectively. It is a pure coincidence that both pairs have almost the same action. Plotted now is the collective contribution of the total number of four orbits in various semiclassical approximations and the exact result. The situation is qualitatively the same as for the tangent bifurcation: both 


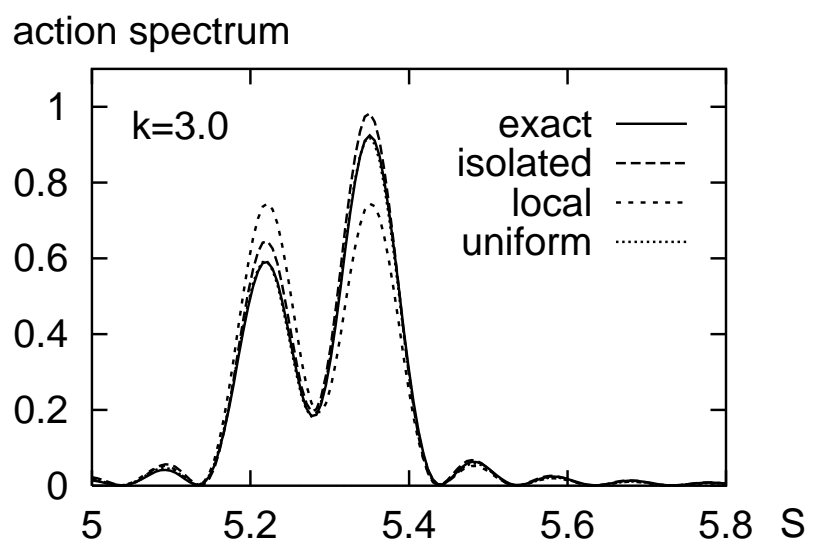

Figure 3. The exact peak in the action spectrum of figure 1 at $k=3.0$ is compared with semiclassically evaluated ones. The uniform approximation works well. The local approximation predicts peaks of equal height. Treating both orbits as isolated gives peaks that are slightly too high.

the local and the uniform approximations are excellent close to the bifurcations; moving away, the uniform approximation remains valid and the sum of isolated orbits starts to work well while the local approximation breaks down.

For period tripling we encounter difficulties from the close neighbourhood of a tangent bifurcation of the satellite. This tangent bifurcation was found to be very close to the tripling for all investigated cases. The problem can be overcome if $j_{\min }$ is chosen large enough in order to ensure that the further satellite which is involved into the tangent bifurcation is well separated, the separation being measured by its difference in action in units of Planck's constant, $\left(j+\frac{1}{2}\right) \Delta S$. Then our analytical expression (24), which does not describe the tangent bifurcation, can be tested without being overshadowed by the existence of the additional satellite. Since the relevant action difference is tiny we raised $j_{\min }$ up to $2^{13}+1$, with $j_{\max }=2^{13}+64$. The result is presented in figure 6 . For the lower values of $k$ the tangent bifurcation at $k=3.525$ is still felt strongly and all semiclassical approximations go wrong. At the period tripling $(k=3.545)$ and beyond it the uniform and the local approximation work well again. The breakdown of the local approximation is observed if one goes to a different scale of the control parameter, as shown in figure 7.

In conclusion, for all cases studied the uniform approximation was found to give excellent results (as long as the results were not overshadowed by the existence of further orbits). The local approximation is of the same quality close to the bifurcation. It breaks down when relations (10) between the monodromy matrices become invalid as one increasingly detunes the control parameter. Along the same line, the action difference of the orbits in units of Planck's constant is large in that region of parameter space, and the use of the sum over isolated orbits (20) makes sense there.

\section{Conclusions}

In this paper we examined semiclassical contributions of three different bifurcations in two-dimensional conservative systems and one-dimensional area-preserving maps. These bifurcations are the generic period- $m$-tupling bifurcations with $m=1,2$ and 3 . We extended the local approximation of de Almeida and Hannay for these cases and derived uniform 
peak height

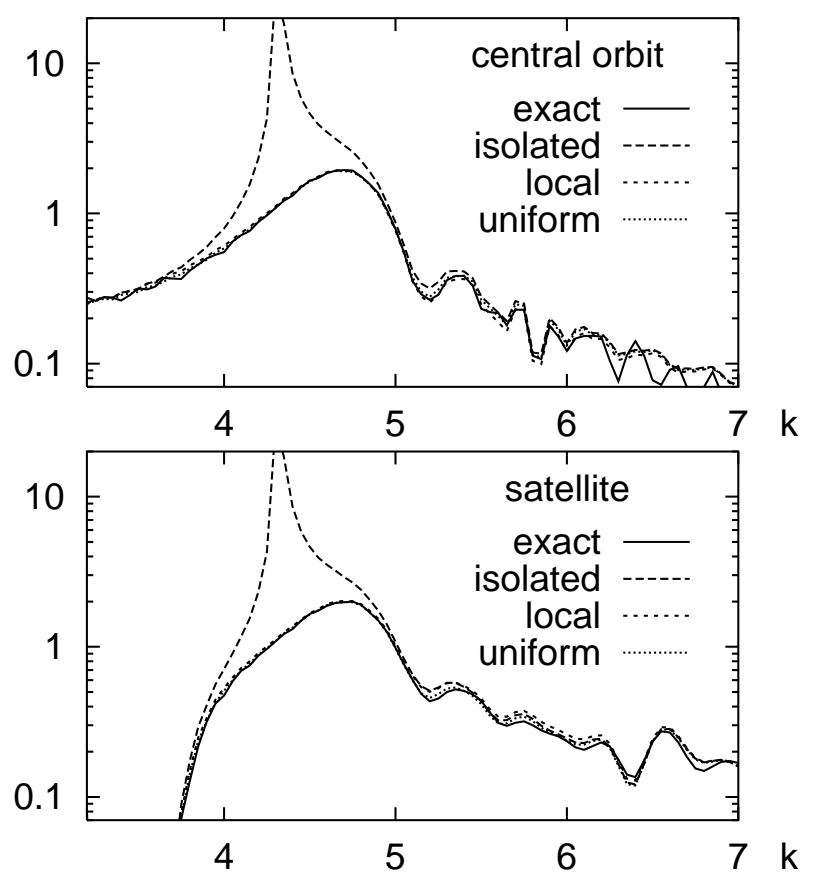

Figure 4. Peak height as in figure 2 for orbits involved in a period doubling bifurcation $(m=2)$ at $k=4.3$. The local and uniform approximations are again accurate before and close to the bifurcation. This time they remain so until overlapping peaks from other orbits give rise to deviations. One such orbit has already been included, others not, as is explained in the text. The sum of individual terms that treats all orbits as isolated fails close to the bifurcation and regains validity far away.

approximations which interpolate over the regime from the bifurcation up to regions where the orbits can be considered isolated. The approximations were tested numerically on the example of a kicked top and were found to be in excellent agreement with exact quantum calculations. The local approximation was found to be good near a bifurcation, but its quality decreased further from a bifurcation. In contrast, the Gutzwiller approximation is good sufficiently far away from a bifurcation, but it diverges at a bifurcation.

We derived the classical properties of periodic orbits that follow from normal forms with higher-order corrections. We further examined the semiclassical contributions of the orbits directly at the bifurcation $(\varepsilon=0)$. It was found that with increasing $m$, bifurcations contribute more strongly to the level density. In more detail, the semiclassical amplitude of the orbits at the bifurcation is of order $\mathcal{O}\left(\hbar^{-7 / 6}\right)$ for $m=1, \mathcal{O}\left(\hbar^{-5 / 4}\right)$ for $m=2$, and $\mathcal{O}\left(\hbar^{-4 / 3}\right)$ for $m=3$. For the case $m \geqslant 5$ it follows from [13] that the semiclassical amplitude at the bifurcation is of order $\mathcal{O}\left(\hbar^{-3 / 2}\right)$. These results are of the general form $\mathcal{O}\left(\hbar^{-1-v}\right)$ where $v$ is the singularity index corresponding to the considered bifurcation [23]. Thus, bifurcations with larger $m$ have a stronger influence on the level density. In addition, for larger $m$ the semiclassical amplitudes decrease more slowly for higher repetitions of the bifurcating periodic orbits. For the $l$ th multiple of the bifurcating orbits the semiclassical amplitude decreases by a factor of $l^{-5 / 6}$ for $m=1$, by $l^{-3 / 4}$ for $m=2$, by $l^{-2 / 3}$ for $m=3$, 


\section{peak height}

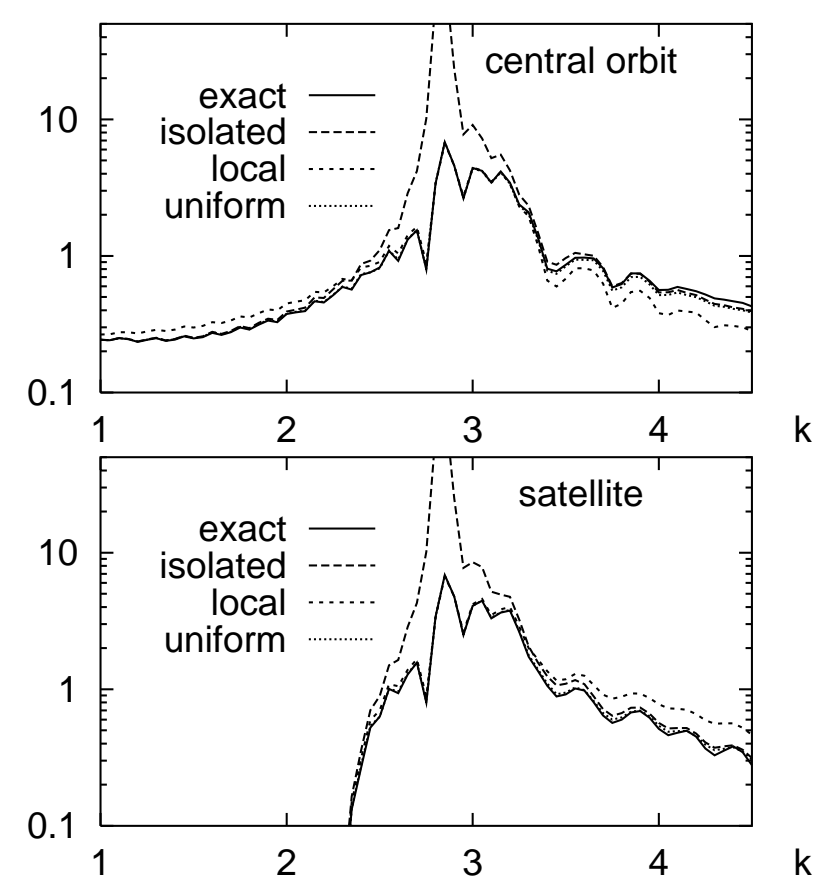

Figure 5. Two pairs of orbits involved in period doublings at $k=2.78$ and $k=2.84$ give rise to overlapping peaks in the action spectrum; the height at the actions of two bifurcating orbits is plotted here. The accuracy of the uniform approximation in the whole parameter range, the failure of the isolated treatment close the bifurcation, and the breakdown of the local approximation at a distance to the bifurcation is clearly visible.

and by $l^{-1 / 2}$ for $m \geqslant 5$. We mention, however, that these results have been obtained by treating the bifurcations isolated from any other bifurcation in the vicinity, an approximation which is expected to break down for large $l$.

In general, all uniform approximations in this paper are only valid as long as the participating periodic orbits do not bifurcate further, i.e. as long as the bifurcations can be considered isolated. If a periodic orbit undergoes several subsequent bifurcations then the uniform approximations have to be modified. In general this is the case if contributions of longer orbits are considered, because they tend to bifurcate more rapidly. The situation thus becomes more and more complex if longer orbits are taken into account. Whether neighbouring orbits or neighbouring groups of orbits can be considered isolated depends on the value of the action difference in comparison with $\hbar$.

An example of a periodic orbit undergoing two subsequent bifurcations was seen in section 5. There a tangent bifurcation occurred very close to a period-tripling bifurcation. Similar situations for period-doubling and period-quadrupling bifurcations have been observed in [24,17]. In these cases the uniform approximation has to be modified by fully integrating over an extended normal form in which higher-order terms are included that describe also subsequent bifurcations. This means that the reduction of the higherorder corrections in the exponent of the oscillatory integral (7), as done in appendices A-C, cannot be performed in these cases. Instead, the higher-order corrections have to be left in 


\section{peak height}

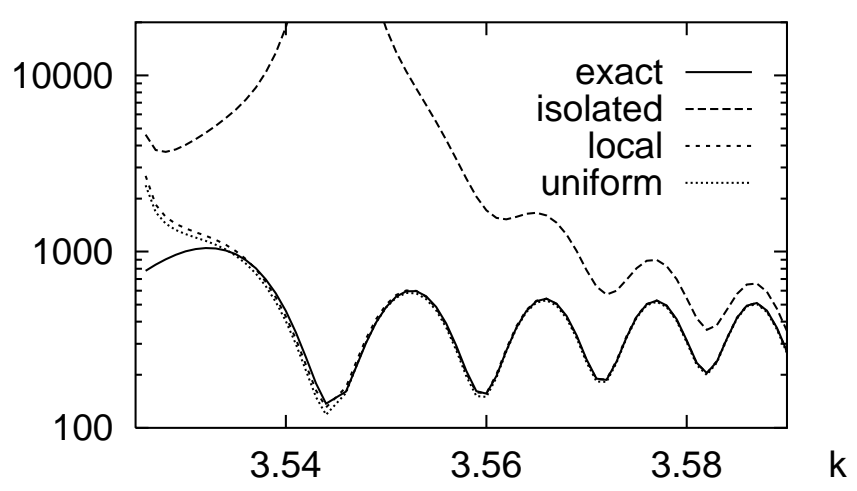

Figure 6. Height of peaks in the action spectrum that arise from orbits involved in a period tripling bifurcation $(m=3)$ at $k=3.545$. The satellite is engaged in a tangent bifurcation with another satellite at $k=3.525$; this makes it necessary to increase $j_{\min }=2^{13}+1$ as discussed in the text. The additional satellite is only felt slightly at the bifurcation and the accuracy is high for the local and uniform approximations.

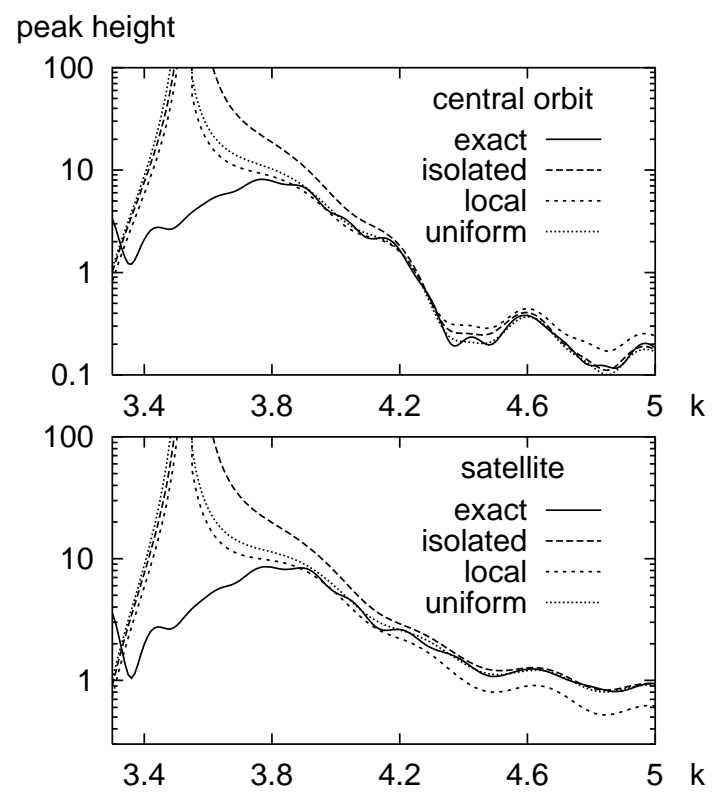

Figure 7. The same period tripling is investigated as in figure 6 . For the chosen $j_{\min }=1$ an additional satellite overshadows the result close to the tripling. As one goes away from the bifurcation to higher $k$ the uniform and isolated approximations work fine and the local approximation is inaccurate.

the exponent. For the mentioned period-tripling bifurcation this was done in [25].

An extension of the present results concerns the case $m=4$, i.e. the period-quadrupling bifurcation. Since the case $m>4$ was treated in [13] this is the only remaining generic bifurcation. The treatment of the generic period-quadrupling bifurcation is more complicated than the other cases since it involves three periodic orbits whose action differences all scale with the same leading power in $\varepsilon$ (for small $\varepsilon$ ). As a consequence one has to add two correction terms to the local approximation in order to obtain a formula which has the correct Gutzwiller limit. Furthermore, the diffraction catastrophe integral for this case can in general not be expressed in terms of simple functions. A treatment of this bifurcation is in preparation. 
A further extension concerns systems with symmetries. In these systems there can be further kinds of bifurcations which are characteristic for the considered symmetry. Normal forms for systems with symmetries have been derived in [26,27]. If these normal forms agree with normal forms for generic bifurcations, then they can be described, with only slight modifications, by the same uniform approximations. For example, in systems with time-reversal and reflection symmetries there can be isochronuous pitchfork bifurcations [27]. These bifurcations are described by formula (15) for period-doubling bifurcations with the replacement of $A_{1}$ by $2 A_{1}$. Another example where the generic formula for $m \geqslant 5$ is applied to symmetric period- $n$-tupling bifurcations with $n=m / 2$ is discussed in [14].

\section{Acknowledgments}

MS would like to thank A Mouchet for helpful discussions, and wishes to acknowledge financial support from the Deutsche Forschungsgemeinschaft under contract no DFG-Ste 241/7-1 and /6-1. HS gratefully acknowledges support from the Sonderforschungsbereich 'Unordnung und große Fluktuationen' of the Deutsche Forschungsgemeinschaft.

\section{Appendix A. Uniform approximation for the isochronuous bifurcation}

The starting point for the derivation of the uniform approximation is the expansion of the generating function in the vicinity of the bifurcation

$$
\hat{S}\left(q^{\prime}, p, E\right)=S_{0}+q^{\prime} p-\varepsilon q^{\prime}-a q^{\prime 3}-b q^{\prime 4}-\frac{\sigma}{2} p^{2} .
$$

Here we went one order higher in $q^{\prime}$ than in the normal form expansion, and in the following we will treat the extra term as a perturbation to obtain the uniform approximation. It is sufficient to consider only corrections in $q^{\prime}$, since the variable $p$ is not essential for the description of the bifurcation. This is expressed by the splitting lemma of catastrophe theory [28]. (See also [29] for higher-order corrections to the normal form for the reduced Hamiltonian.)

By rescaling $q^{\prime}$ and $p$ it can always be achieved that $\sigma= \pm 1$ in (27). The fixed points of the map generated by $\hat{S}$ are given by $\hat{S}_{q^{\prime}}=p, \hat{S}_{p}=q^{\prime}$, and up to second order in $\sqrt{|\varepsilon|}$ we get for the two fixed points engaged in the bifurcation

$$
\begin{aligned}
& p_{1,2}=0 \\
& q_{1,2}^{\prime}= \pm \sqrt{-\frac{\varepsilon}{3 a}}+\frac{2 b \varepsilon}{9 a^{2}}+\mathcal{O}\left(|\varepsilon|^{3 / 2}\right)
\end{aligned}
$$

while we will not consider the additional fixed point which now formally arises as a third solution of the equations. The value of the actions $S=\hat{S}-q^{\prime} p$ at the fixed points is

$$
S_{1,2}=S_{0} \mp \frac{2 \varepsilon}{3} \sqrt{-\frac{\varepsilon}{3 a}}-\frac{b \varepsilon^{2}}{9 a^{2}}+\mathcal{O}\left(|\varepsilon|^{5 / 2}\right)
$$

where $S_{0}$ is the value of the generating function at the origin. If $\varepsilon$ has the same sign as $a$ then the orbits are complex and there is an ambiguity for choosing the sign of the square root in equations (28) and (29). We then choose to give the index 1 to that orbit for which the imaginary part of the action is positive. This formally corresponds to choosing $\sqrt{-\varepsilon /(3 a)}=-\mathrm{i} \operatorname{sign}(\varepsilon) \sqrt{|\varepsilon /(3 a)|}$. 
The periods of the orbits are given by

$$
T_{1,2}=T_{0} \mp \varepsilon_{E} \sqrt{\frac{-\varepsilon}{3 a}}+\mathcal{O}(\varepsilon)
$$

where $T_{0}=\partial \hat{S} / \partial E$, evaluated at the origin, and corresponds to the mean period. The traces of the monodromy matrices follow from the relation

$$
\operatorname{Tr} M=\left(\frac{\partial^{2} \hat{S}}{\partial p \partial q^{\prime}}\right)^{-1}\left(1+\frac{\partial^{2} \hat{S}}{\partial p \partial q^{\prime}} \frac{\partial^{2} \hat{S}}{\partial p \partial q^{\prime}}-\frac{\partial^{2} \hat{S}}{\partial p^{2}} \frac{\partial^{2} \hat{S}}{\partial q^{\prime 2}}\right)
$$

which is evaluated at the stationary points and leads to

$$
\operatorname{Tr} M_{1,2}=2 \mp \sigma 6 a \sqrt{-\frac{\varepsilon}{3 a}}+\frac{8 \sigma b}{3 a} \varepsilon+\mathcal{O}\left(|\varepsilon|^{3 / 2}\right) .
$$

Furthermore the semiclassical amplitudes are given by

$$
A_{1,2}=\frac{T_{1,2}}{l \sqrt{\eta_{1,2}\left(\operatorname{Tr} M_{1,2}-2\right)}}=\frac{1}{l|12 a \varepsilon|^{1 / 4}}\left(T_{0} \mp\left(\frac{2 b}{3 a} T_{0}+\varepsilon_{E}\right) \sqrt{-\frac{\varepsilon}{3 a}}+\mathcal{O}(\varepsilon)\right)
$$

where $l=r_{1}=r_{2}$ is the repetition number of the orbits, $\eta_{i}=\operatorname{sign}\left(\operatorname{Re}\left(\operatorname{Tr} M_{i}-2\right)\right)$ when the orbits are real and $\eta_{i}=-\mathrm{i} \operatorname{sign}\left(\operatorname{Im}\left(\operatorname{Tr} M_{i}\right)\right)$ when they are complex.

In the following we will use the definitions

$$
\begin{array}{ccc}
\bar{S}=\frac{S_{1}+S_{2}}{2} & \Delta S=\frac{S_{1}-S_{2}}{2} & \bar{A}=\frac{A_{1}+A_{2}}{2} \\
\Delta A=\frac{A_{1}-A_{2}}{2} & \bar{v}=\frac{\nu_{1}+\nu_{2}}{2} &
\end{array}
$$

where $v_{1}$ and $v_{2}$ are the Maslov indices of the orbits when they are real.

We continue now with the evaluation of the integral in (7). The main contribution to the integral over $q^{\prime}$ comes from the region near the stationary points. For that reason we consider $q^{\prime}$ in the following as a quantity of order $\mathcal{O}\left(|\varepsilon|^{1 / 2}\right)$. Then the exponent of the integral can be simplified by substituting

$$
q^{\prime}=\frac{\varepsilon b}{9 a^{2}}+x-x^{2} \frac{b}{3 a}
$$

which leads to a reduction of the generating function

$$
\hat{S}\left(q^{\prime}, p, E\right)-q^{\prime} p=\bar{S}-\varepsilon x-a x^{3}-\frac{\sigma}{2} p^{\prime 2}+\mathcal{O}\left(|\varepsilon|^{5 / 2}\right) .
$$

This is just the usual normal form with the replacement of $S_{0}$ by $\bar{S}$. Furthermore, the exponential prefactor in the integral is modified by the Jacobian of the transformation. After an integration over $p$ we obtain

$$
\begin{aligned}
d_{\xi}(E)=\operatorname{Re}\left[\frac{\exp \left(\frac{\mathrm{i}}{\hbar} \bar{S}-\frac{\mathrm{i} \pi}{2} \nu-\frac{\mathrm{i} \pi}{4} \sigma\right)}{l \sqrt{2 \pi^{3} \hbar^{3}}} \int_{-\infty}^{\infty} \mathrm{d} x\left(T_{0}-\frac{2 b}{3 a} T_{0} x-\varepsilon_{E} x\right)\right. \\
\left.\quad \times \exp \left\{-\frac{\mathrm{i}}{\hbar}\left(\varepsilon x+a x^{3}\right)\right\}\right]
\end{aligned}
$$

where the exponential prefactor has been expanded up to $\mathcal{O}(x)$. The integral in (37) can be split into two terms. The first term with the constant term in the exponential prefactor has the same form as the local approximation, and the second term is proportional to the derivative of the first term with respect to $\varepsilon$. The integrals can be found in the section on Airy functions in [30]. The result depends on whether $\varepsilon$ has the same or the opposite sign 
as $a$. We first consider the case when they have opposite sign, i.e. when the orbits are real. The contribution to the level density is then given by

$$
\begin{gathered}
d_{\xi}(E)=\operatorname{Re} \frac{\exp \left(\frac{\mathrm{i}}{\hbar} \bar{S}-\frac{\mathrm{i} \pi}{2} \nu-\frac{\mathrm{i} \pi}{4} \sigma\right)}{l \sqrt{2 \pi^{3} \hbar^{3}}}\left\{\frac{2 \pi T_{0}}{3} \sqrt{\left|\frac{\varepsilon}{3 a}\right|}\left(J_{-1 / 3}\left(\frac{|\Delta S|}{\hbar}\right)+J_{1 / 3}\left(\frac{|\Delta S|}{\hbar}\right)\right)\right. \\
\left.-\frac{2 \pi \mathrm{i} \varepsilon}{9|a|}\left(\varepsilon_{E}+\frac{2 b T_{0}}{3 a}\right)\left(J_{-2 / 3}\left(\frac{|\Delta S|}{\hbar}\right)-J_{2 / 3}\left(\frac{|\Delta S|}{\hbar}\right)\right)\right\} .
\end{gathered}
$$

This result can be expressed also by the Airy function Ai and its derivative, but it seems more natural to express it by Bessel functions since then the analogy to the results for higher repetition numbers is more visible. All coefficients can be expressed by the classical actions and amplitudes. The final result is

$$
\begin{aligned}
d_{\xi}(E)=\frac{1}{\pi \hbar} \mid & \left.\frac{2 \pi \Delta S}{3 \hbar}\right|^{1 / 2}\left\{\bar{A} \cos \left(\frac{\bar{S}}{\hbar}-\frac{\pi}{2} \bar{v}\right)\left(J_{-1 / 3}\left(\frac{|\Delta S|}{\hbar}\right)+J_{1 / 3}\left(\frac{|\Delta S|}{\hbar}\right)\right)\right. \\
& \left.\quad-\operatorname{sign}(\Delta S) \Delta A \cos \left(\frac{\bar{S}}{\hbar}-\frac{\pi}{2}(\bar{v}-1)\right)\left(J_{-2 / 3}\left(\frac{|\Delta S|}{\hbar}\right)-J_{2 / 3}\left(\frac{|\Delta S|}{\hbar}\right)\right)\right\} .
\end{aligned}
$$

Here we replaced $\nu+\sigma / 2$ by the mean Maslov index $\bar{v}$. It can be seen that this is correct from a stationary phase evaluation of the integrals which shows the $v_{1,2}=v+\sigma / 2 \pm \operatorname{sign}(\Delta S) / 2$. The first term in the curly brackets in (39) is just the local approximation in which the stability factors of both orbits are equal. At the bifurcation $(\varepsilon \rightarrow 0)$ the dependence of the classical quantities on $\varepsilon$ guarantees a finite result. The second term in the curly brackets is the uniform correction which ensures the correct limit as $\hbar \rightarrow 0$ for finite $\varepsilon$, since then one arrives at a sum of two individual contributions for the two orbits, each of Gutzwiller's type.

On the other side of the bifurcation where the orbits are complex and $\varepsilon$ and $a$ have the same sign, the evaluation of the integral in (37) leads to

$$
\begin{aligned}
d_{\xi}(E)=\operatorname{Re} \frac{\exp \left(\frac{\mathrm{i}}{\hbar} \bar{S}-\frac{\mathrm{i} \pi}{2} \nu-\frac{\mathrm{i} \pi}{4} \sigma\right)}{l \sqrt{2 \pi^{3} \hbar^{3}}} \\
\quad \times\left\{\frac{2 T_{0}}{3} \sqrt{\left|\frac{\varepsilon}{a}\right|} K_{1 / 3}\left(\frac{|\Delta S|}{\hbar}\right)+\frac{2 \mathrm{i} \varepsilon}{3 \sqrt{3}|a|}\left(\varepsilon_{E}+\frac{2 b T_{0}}{3 a}\right) K_{2 / 3}\left(\frac{|\Delta S|}{\hbar}\right)\right\} .
\end{aligned}
$$

In this equation the mean quantities $\bar{S}$ and $\bar{A}$ are real, but the differences $\Delta S$ and $\Delta A$ are purely imaginary. The quantities are defined in equations (29), (33) and (34) with the convention for the sign of the square root that has been discussed above

$$
\begin{aligned}
d_{\xi}(E)=\operatorname{Re} \frac{1}{\pi \hbar}\left|\frac{2 \Delta S}{\pi \hbar}\right|^{1 / 2} \exp \left(\frac{\mathrm{i}}{\hbar} \bar{S}-\frac{\mathrm{i} \pi}{2} v-\frac{\mathrm{i} \pi}{4} \sigma\right) \\
\times\left\{\bar{A} K_{1 / 3}\left(\frac{|\Delta S|}{\hbar}\right)+\Delta A K_{2 / 3}\left(\frac{|\Delta S|}{\hbar}\right)\right\} .
\end{aligned}
$$

In the limit that the argument of the $K$-Bessel functions is large one obtains only the contribution of that orbit for which the imaginary part of the action is positive

$$
d_{\xi}(E)=\operatorname{Re} \frac{A_{1}}{\pi \hbar} \exp \left(\frac{\mathrm{i}}{\hbar} S_{1}-\frac{\mathrm{i} \pi}{2} v-\frac{\mathrm{i} \pi}{4} \sigma\right) .
$$

This is connected to Stokes' phenomenon. 
We now discuss in slightly more detail the approximation that was applied in this section. We started with the extended normal form and derived approximations (38) and (40) from it. The step to obtain the uniform approximation consisted of expressing the coefficients of the normal forms in these equations by the classical actions and amplitudes of the orbits. This resulted in equations (39) and (41), which have the correct asymptotic form as $\delta S / \hbar$, becoming large. These approximations thus have the properties of uniform approximations, they are valid close to the bifurcation as well as far away from it. From another point of view, they are valid in both limits $\hbar \rightarrow 0$ as well as $\varepsilon \rightarrow 0$. One might ask what would happen if one added even more corrections to the normal form (27). After a modification of transformation (35) this leads to higher powers of $x$ in the exponential prefactor in integral (37). This results in higher derivatives of the Airy function which can be expressed by the Airy function and its first derivative (by using the differential equation for the Airy function). After expressing the coefficients by the classical actions and amplitudes, one obtains exactly the same equations (39) and (41) since the coefficients of the Airy functions are fixed by the asymptotic form of the equations for large $\delta S / \hbar$. The only difference in comparison with the previous derivation is that the expansions of the classical actions and amplitudes are carried out in higher order.

\section{Appendix B. Uniform approximation for the period-doubling bifurcation}

For the period-doubling bifurcation, we start again with the normal form $\hat{S}\left(q^{\prime}, p, E\right)$ of the action and go beyond the local approximation by incorporating higher-order terms. As in the case $m=1$ it is sufficient to consider only corrections in $q^{\prime}$. The extended normal form is then

$$
\hat{S}\left(q^{\prime}, p, E\right)=S_{0}+q^{\prime} p-\varepsilon q^{\prime 2}-a q^{\prime 4}-b q^{\prime 6}-\frac{\sigma}{2} p^{2}
$$

where once more $\sigma= \pm 1$. No odd powers of $q^{\prime}$ appear since both satellite fixed points belong to the same orbit and have the same action and stability. The fixed points lie at

$$
\begin{array}{ll}
p_{0}=0 & q_{0}^{\prime}=0 \\
p_{1}=0 & q_{1}^{\prime}= \pm \sqrt{-\frac{\varepsilon}{2 a}}\left(1+\frac{3 b \varepsilon}{8 a^{2}}\right)+\mathcal{O}\left(|\varepsilon|^{5 / 2}\right)
\end{array}
$$

where the subscript 0 indicates the central orbit which has a repetition number of $2 l$, and the subscript 1 denotes the satellite orbit. The actions of the orbits are $S_{0}$ and

$$
S_{1}=S_{0}+\frac{\varepsilon^{2}}{4 a}+\frac{b \varepsilon^{3}}{8 a^{3}}+\mathcal{O}\left(\varepsilon^{4}\right)
$$

and the periods are given by $T_{0}=\partial \hat{S} / \partial E$ evaluated at the origin and

$$
T_{1}=T_{0}+\frac{\varepsilon_{E}}{2 a} \varepsilon+\mathcal{O}\left(\varepsilon^{2}\right)
$$

The traces of the monodromy matrix follow from (31) and are given by

$$
\begin{aligned}
& \operatorname{Tr} M_{0}=2-2 \sigma \varepsilon \\
& \operatorname{Tr} M_{1}=2+4 \sigma \varepsilon-\frac{3 b \sigma}{a^{2}} \varepsilon^{2}+\mathcal{O}\left(\varepsilon^{3}\right)
\end{aligned}
$$

and the stability prefactors follow as

$$
\begin{aligned}
& A_{0}=\left|8 \varepsilon l^{2}\right|^{-1 / 2} T_{0} \\
& A_{1}=\left|4 \varepsilon l^{2}\right|^{-1 / 2}\left(T_{0}+\frac{\varepsilon_{E}}{2 a} \varepsilon+\frac{3 b T_{0}}{8 a^{2}} \varepsilon+\mathcal{O}\left(\varepsilon^{2}\right)\right)
\end{aligned}
$$


where the repetition numbers of the orbits are $r_{0}=2 l$ and $r_{1}=l$.

In the following we will use the definitions

$$
\bar{S}=\frac{S_{1}+S_{0}}{2} \quad \Delta S=\frac{S_{1}-S_{0}}{2} \quad \bar{v}=\frac{v_{1}+v_{0}}{2} .
$$

In order to evaluate integral (7) we want to get rid of the dependence of the exponent on $q^{\prime 6}$ and introduce for this purpose a new variable by

$$
q^{\prime 2}=x^{2}-x^{4} \frac{b}{2 a} \text {. }
$$

If one considers $q^{\prime}$ as a quantity of order $\mathcal{O}\left(|\varepsilon|^{1 / 2}\right)$ the generating function reduces to

$$
\hat{S}-q^{\prime} p=S_{0}-\varepsilon x^{2}-\tilde{a} x^{4}-\frac{\sigma}{2} p^{2}+\mathcal{O}\left(\varepsilon^{4}\right)
$$

where $\tilde{a}=a-b \varepsilon /(2 a)$. One can check that the new prefactor in front of $x^{4}$ yields the correct action (45) up to order $\varepsilon^{3}$ at the fixed points. After integrating over $p$ one arrives at

$$
\begin{aligned}
d_{\xi}(E)=\operatorname{Re}[ & \frac{\exp \left(\frac{\mathrm{i}}{\hbar} S_{0}-\frac{\mathrm{i} \pi}{2} \nu-\frac{\mathrm{i} \pi}{4} \sigma\right)}{l(2 \pi \hbar)^{3 / 2}} \int_{0}^{\infty} \mathrm{d} x\left(T_{0}-\frac{3 b T_{0}}{4 a} x^{2}-\varepsilon_{E} x^{2}\right) \\
& \left.\times \exp \left\{-\frac{\mathrm{i}}{\hbar}\left(\varepsilon x^{2}+\tilde{a} x^{4}\right)\right\}\right]
\end{aligned}
$$

where the exponential prefactor has been expanded up to order $\mathcal{O}\left(x^{2}\right)$. Once more the integral splits into two terms of type

$$
\begin{array}{rl}
B_{1}=\int_{-\infty}^{+\infty} \mathrm{d} & x \exp \left(-\frac{\mathrm{i}}{\hbar}\left(\varepsilon x^{2}+\tilde{a} x^{4}\right)\right) \\
= & \frac{\pi}{2}\left|\frac{\varepsilon}{2 \tilde{a}}\right|^{1 / 2} \exp \left\{\frac{\mathrm{i}}{\hbar} \Delta S\right\}\left(J_{-1 / 4}\left(\left|\frac{\Delta S}{\hbar}\right|\right) \mathrm{e}^{-\mathrm{i} \sigma_{1} \pi / 8}-\sigma_{1} \tilde{\sigma}_{2} J_{1 / 4}\left(\left|\frac{\Delta S}{\hbar}\right|\right) \mathrm{e}^{\mathrm{i} \sigma_{1} \pi / 8}\right)
\end{array}
$$

and

$$
\begin{aligned}
B_{2}=\int_{-\infty}^{+\infty} \mathrm{d} x & x^{2} \exp \left(-\frac{\mathrm{i}}{\hbar}\left(\varepsilon x^{2}+\tilde{a} x^{4}\right)\right) \\
= & -\frac{\varepsilon}{4 \tilde{a}} B_{1}-\frac{\pi \varepsilon}{8 \tilde{a}}\left|\frac{\varepsilon}{2 \tilde{a}}\right|^{1 / 2} \\
& \times \exp \left\{\frac{\mathrm{i}}{\hbar} \Delta S\right\}\left(J_{3 / 4}\left(\left|\frac{\Delta S}{\hbar}\right|\right) \mathrm{e}^{\mathrm{i} \sigma_{1} \pi 3 / 8}-\sigma_{1} \tilde{\sigma}_{2} J_{-3 / 4}\left(\left|\frac{\Delta S}{\hbar}\right|\right) \mathrm{e}^{-\mathrm{i} \sigma_{1} \pi 3 / 8}\right)
\end{aligned}
$$

where $\Delta S$ as given above, $\sigma_{1}=\operatorname{sign}(\tilde{a})=\operatorname{sign}(\Delta S)$, and $\tilde{\sigma}_{2}=\operatorname{sign}(\varepsilon)$. Now we introduce $\sigma_{2}=-\tilde{\sigma}_{1} \tilde{\sigma}_{2}$ to discriminate between both sides of the bifurcation $\left(\sigma_{2}=1\right.$ when the satellite orbit is real and $\sigma_{2}=-1$ when it is complex). Expressing all coefficients by the actions $S_{0,1}$ and stability factors $A_{0,1}$ the final result is

$$
\begin{aligned}
d_{\xi}(E)=\operatorname{Re} \frac{1}{\pi \hbar}\left|\frac{\pi \Delta S}{2 \hbar}\right|^{1 / 2} \exp \left(\frac{\mathrm{i}}{\hbar} \bar{S}-\frac{\mathrm{i} \pi}{2} v-\frac{\mathrm{i} \pi}{4} \sigma\right) \\
\times\left\{\left(\frac{A_{1}}{2}+\frac{A_{0}}{\sqrt{2}}\right)\left(\sigma_{2} J_{1 / 4}\left(\frac{|\Delta S|}{\hbar}\right) \mathrm{e}^{\mathrm{i} \sigma_{1} \pi / 8}+J_{-1 / 4}\left(\frac{|\Delta S|}{\hbar}\right) \mathrm{e}^{-\mathrm{i} \sigma_{1} \pi / 8}\right)\right. \\
\left.+\left(\frac{A_{1}}{2}-\frac{A_{0}}{\sqrt{2}}\right)\left(J_{3 / 4}\left(\frac{|\Delta S|}{\hbar}\right) \mathrm{e}^{\mathrm{i} \sigma_{1} 3 \pi / 8}+\sigma_{2} J_{-3 / 4}\left(\frac{|\Delta S|}{\hbar}\right) \mathrm{e}^{-\mathrm{i} \sigma_{1} 3 \pi / 8}\right)\right\} .
\end{aligned}
$$


In contrast to the tangent bifurcation, all appearing classical quantities are real even when the satellite orbit is complex. In the limit where the argument of the Bessel functions is large, the expression reduces to a sum of the two Gutzwiller contributions of the orbits when $\sigma_{2}=1$, and to the single Gutzwiller contribution of the central orbit when $\sigma_{2}=-1$.

\section{Appendix C. Uniform approximation for the period-tripling bifurcation}

For the period-tripling bifurcation the calculations are more involved, since the orbits which participate in the bifurcation now lie in a plane. For the previous two bifurcations ( $m=1$ and $m=2$ ) the orbits lay on a line so that one has only got to treat one-dimensional integrals.

The normal form of the generating function $\hat{S}\left(q^{\prime}, p, E\right)$ in terms of $q^{\prime}$ - and $p$-coordinates is given by

$$
\hat{S}\left(p, q^{\prime}, E\right)=S_{0}+p q^{\prime}-\frac{\varepsilon}{2}\left(p^{2}+q^{\prime 2}\right)-\frac{a}{2 \sqrt{2}}\left(p^{3}-3 p q^{\prime 2}\right) .
$$

The function $\hat{S}\left(q^{\prime}, p, E\right)-q^{\prime} p$ has four stationary points, one at the origin which corresponds to the central orbit, and three others which lie on an equilateral triangle and correspond to the satellite orbit. In contrast to the previous two cases, one has to go two orders beyond the normal form in the expansion of the generating function $\hat{S}$ since the first correction does not change the amplitude prefactor of the satellite orbit. The higher-order terms in this expansion are restricted by the condition that the three stationary points which correspond to the satellite orbit have to yield all the same three action and the same trace of the monodromy matrix of the satellite orbit. This leads to the expansion

$$
\begin{aligned}
\hat{S}\left(p, q^{\prime}, E\right)= & S_{0}+p q^{\prime}-\frac{\varepsilon}{2}\left(p^{2}+q^{\prime 2}\right)-\frac{a}{2 \sqrt{2}}\left(p^{3}-3 p q^{\prime 2}\right)-\frac{b}{4}\left(p^{4}+2 p^{2} q^{\prime 2}+q^{\prime 4}\right) \\
& -\frac{9 a^{2}}{8} p\left(3 p^{2} q^{\prime}-q^{\prime 3}\right)-\frac{c}{4 \sqrt{2}}\left(p^{5}-2 p^{3} q^{\prime 2}-3 p q^{\prime 4}\right) \\
& -\frac{27 a^{3}}{64 \sqrt{2}}\left(39 p^{3} q^{\prime 2}-29 p q^{\prime 4}\right)-\frac{3 a b}{4 \sqrt{2}}\left(-3 p^{4} q^{\prime}+p^{2} q^{\prime 3}\right) .
\end{aligned}
$$

The requirement that the three stationary points yield the same classical properties also allows a further term of the form $d q^{\prime}\left(p^{2}+q^{\prime 2}\right)\left(3 p^{2}-q^{\prime 2}\right)$, but the properties of the orbits do not depend on this term and it can be removed in a later step by modifying the coefficients (63) of the transformation (62). For that reason we do not include it.

In the following the expansions of several quantities in terms of $\varepsilon$ are given. The action of the satellite orbit is

$$
S_{1}=S_{0}-\frac{4}{27 a^{2}} \varepsilon^{3}-\frac{16 b}{81 a^{4}} \varepsilon^{4}-\frac{4\left(64 b^{2}-243 a^{4}-24 a c\right)}{729 a^{6}} \varepsilon^{5}+\mathcal{O}\left(\varepsilon^{6}\right)
$$

and its period is of the form

$$
T_{1}=T_{0}+\left.\frac{\partial \hat{S}}{\partial E}\right|_{p=p_{1}, q^{\prime}=q_{1}^{\prime}}=T_{0}-\frac{4 \varepsilon_{E}}{9 a^{2}} \varepsilon^{2}+\mathcal{O}\left(\varepsilon^{3}\right) .
$$

The traces of the stability matrices of the periodic orbits follow from (31) and are given by

$$
\begin{aligned}
& \operatorname{Tr} M_{0}=2-\varepsilon^{2} \\
& \operatorname{Tr} M_{1}=2+3 \varepsilon^{2}+\frac{\left(2511 a^{4}-128 b^{2}+192 a c\right)}{54 a^{4}} \varepsilon^{4}+\mathcal{O}\left(\varepsilon^{5}\right) .
\end{aligned}
$$


The amplitude prefactors follow as

$$
\begin{aligned}
A_{0}= & \frac{T_{0}}{3 l \sqrt{\left|\operatorname{Tr} M_{0}-2\right|}}=\frac{T_{0}}{3 l|\varepsilon|} \\
A_{1}= & \frac{T_{1}}{l \sqrt{\left|\operatorname{Tr} M_{1}-2\right|}}=\frac{1}{\sqrt{3} l|\varepsilon|} \\
& \quad \times\left(T_{0}-\frac{4 \varepsilon_{E}}{9 a^{2}} \varepsilon^{2}-\frac{T_{0}\left(2511 a^{4}-128 b^{2}+192 a c\right)}{324 a^{4}} \varepsilon^{2}\right)+\mathcal{O}\left(\varepsilon^{2}\right)
\end{aligned}
$$

where the repetition numbers of the orbits are $r_{0}=3 l$ and $r_{1}=l$.

We continue now with the evaluation of the integrals in (7) with the generating function of (57). The exponent in the integral is simplified by applying a transformation of the form

$$
\begin{aligned}
& p=\tilde{p}+c_{1} \tilde{p}^{2}+c_{2} \tilde{p} \tilde{q}+c_{3} \tilde{q}^{2}+c_{4} \tilde{p}^{3}+c_{5} \tilde{p}^{2} \tilde{q}+c_{6} \tilde{p} \tilde{q}^{2}+c_{7} \tilde{q}^{3} \\
& q^{\prime}=\tilde{q}+d_{1} \tilde{p}^{2}+d_{2} \tilde{p} \tilde{q}+d_{3} \tilde{q}^{2}+d_{4} \tilde{p}^{3}+d_{5} \tilde{p}^{2} \tilde{q}+d_{6} \tilde{p} \tilde{q}^{2}+d_{7} \tilde{q}^{3}
\end{aligned}
$$

which removes the terms proportional to $q^{\prime n} p^{4-n}$ and $q^{\prime n} p^{5-n}$ in the exponent (within the considered order of the approximation). The coefficients of the transformation are given by

$$
\begin{aligned}
& c_{1}=-\frac{b \sqrt{2}}{6 a}-\frac{\sqrt{2}}{216 a^{3}}\left(28 b^{2}-243 a^{4}-24 a c\right) \varepsilon \quad c_{2}=-\frac{3 \sqrt{2} a}{4} \quad c_{3}=-c_{1} \\
& c_{4}=-\frac{27 a^{4}-2 b^{2}+2 a c}{12 a^{2}} \quad c_{5}=\frac{b}{4} \\
& c_{6}=-\frac{351 a^{4}-16 b^{2}+16 a c}{96 a^{2}} \quad c_{7}=-\frac{b}{4} \\
& d_{1}=\frac{3 \sqrt{2} a}{4} \quad d_{2}=-2 c_{1} \quad d_{3}=0 \quad d_{4}=-b \\
& d_{5}=-\frac{27 a^{4}-16 b^{2}+16 a c}{96 a^{2}} \quad d_{6}=\frac{b}{2} \quad d_{7}=-\frac{81 a^{4}-8 b^{2}+8 a c}{48 a^{2}}
\end{aligned}
$$

and the transformation leads to

$$
\begin{aligned}
d_{\xi}(E)=\frac{1}{6 l \pi^{2} \hbar^{2}} & \operatorname{Re} \int_{-\infty}^{\infty} \mathrm{d} \tilde{p} \int_{-\infty}^{\infty} \mathrm{d} \tilde{q}\left[\alpha_{1}+\alpha_{2}\left(\tilde{p}^{2}+\tilde{q}^{2}\right)\right] \\
& \times \exp \left\{\frac{\mathrm{i}}{\hbar}\left(S_{0}-\frac{\varepsilon}{2}\left(\tilde{p}^{2}+\tilde{q}^{2}\right)-\frac{\tilde{a}}{2 \sqrt{2}}\left(\tilde{p}^{3}-3 \tilde{p} \tilde{q}^{2}\right)\right)-\frac{\mathrm{i} \pi}{2} v\right\}
\end{aligned}
$$

where the exponential prefactor in (7) times the Jacobian of the transformation (62) has been expanded up to order $\varepsilon^{2}$. The new constants appearing in (64) are

$\alpha_{1}=T_{0}$

$\alpha_{2}=-\frac{144 \varepsilon_{E} a^{2}+2511 T_{0} a^{4}-128 T_{0} b^{2}+192 T_{0} a c}{288 a^{2}}=\frac{27 a^{2} l}{4|\varepsilon|}\left(\frac{A_{1}}{2 \sqrt{3}}-\frac{A_{0}}{2}\right)$

$\tilde{a}=a-\frac{2 b}{3 a} \varepsilon+\frac{243 a^{4}-28 b^{2}+24 a c}{54 a^{3}} \varepsilon^{2}$.

After a change of variables $\tilde{p}=\sqrt{2 I} \cos \Phi$ and $\tilde{q}=\sqrt{2 I} \sin \Phi$ the integrals in (64) are transformed into

$$
\begin{aligned}
d_{\xi}(E)=\frac{1}{6 l \pi^{2} \hbar^{2}} & \operatorname{Re} \int_{0}^{\infty} \mathrm{d} I \int_{0}^{2 \pi} \mathrm{d} \Phi\left[\alpha_{1}+2 \alpha_{2} I\right] \\
& \times \exp \left\{\frac{\mathrm{i}}{\hbar}\left(S_{0}-\varepsilon I-\tilde{a} I^{3 / 2} \cos (3 \Phi)\right)-\frac{\mathrm{i} \pi}{2} \nu\right\}
\end{aligned}
$$




$$
=\frac{1}{3 l \pi \hbar^{2}} \operatorname{Re} \int_{0}^{\infty} \mathrm{d} I\left[\alpha_{1}+2 \alpha_{2} I\right] J_{0}\left(\frac{\tilde{a} I^{3 / 2}}{\hbar}\right) \exp \left\{\frac{\mathrm{i}}{\hbar}\left(S_{0}-\varepsilon I\right)-\frac{\mathrm{i} \pi}{2} v\right\}
$$

where the relation

$$
\int_{0}^{2 \pi} \mathrm{d} \Phi \exp \{\mathrm{i} z \cos (m \Phi)\}=2 \pi J_{0}(z)
$$

has been used. The two remaining integrals are evaluated according to appendix D and result in

$$
\begin{gathered}
\int_{0}^{\infty} \mathrm{d} I J_{0}\left(\frac{\tilde{a} I^{3 / 2}}{\hbar}\right) \exp \left\{-\frac{\mathrm{i}}{\hbar} \varepsilon I\right\}=\frac{\hbar}{|\varepsilon|} \sqrt{\frac{2 \pi|\Delta S|}{\hbar}} \exp \left\{\frac{\mathrm{i} \Delta S}{\hbar}\right\} \\
\times\left[J_{-1 / 6}\left(\frac{|\Delta S|}{\hbar}\right)+\mathrm{i} \sigma J_{1 / 6}\left(\frac{|\Delta S|}{\hbar}\right)\right]
\end{gathered}
$$

and

$$
\begin{aligned}
\int_{0}^{\infty} \mathrm{d} I I J_{0}( & \left.\frac{\tilde{a} I^{3 / 2}}{\hbar}\right) \exp \left\{-\frac{\mathrm{i}}{\hbar} \varepsilon I\right\}=\frac{2 \hbar \varepsilon^{2}}{9|\varepsilon| \tilde{a}^{2}} \sqrt{\frac{2 \pi|\Delta S|}{\hbar}} \exp \left\{\frac{\mathrm{i} \Delta S}{\hbar}\right\} \\
\times & {\left[J_{-1 / 6}\left(\frac{|\Delta S|}{\hbar}\right)+\mathrm{i} \sigma J_{1 / 6}\left(\frac{|\Delta S|}{\hbar}\right)+J_{-5 / 6}\left(\frac{|\Delta S|}{\hbar}\right)+\mathrm{i} \sigma J_{5 / 6}\left(\frac{|\Delta S|}{\hbar}\right)\right] }
\end{aligned}
$$

where $\Delta S=\left(S_{1}-S_{0}\right) / 2=-2 \varepsilon^{3} /\left(27 \tilde{a}^{2}\right)$ and $\sigma=\operatorname{sign}(\Delta S)=-\operatorname{sign}(\varepsilon)$. Altogether one obtains

$$
\begin{aligned}
d_{\xi}(E)=\frac{1}{\pi \hbar} & \operatorname{Re} \sqrt{\frac{2 \pi|\Delta S|}{\hbar}} \exp \left\{\frac{\mathrm{i}}{\hbar} \bar{S}-\frac{\mathrm{i} \pi}{2} v\right\} \\
& \times\left\{\left(\frac{A_{0}}{2}+\frac{A_{1}}{2 \sqrt{3}}\right)\left[J_{-1 / 6}\left(\frac{|\Delta S|}{\hbar}\right)+\mathrm{i} \sigma J_{1 / 6}\left(\frac{|\Delta S|}{\hbar}\right)\right]\right. \\
& \left.-\left(\frac{A_{0}}{2}-\frac{A_{1}}{2 \sqrt{3}}\right)\left[J_{-5 / 6}\left(\frac{|\Delta S|}{\hbar}\right)+\mathrm{i} \sigma J_{5 / 6}\left(\frac{|\Delta S|}{\hbar}\right)\right]\right\}
\end{aligned}
$$

where $\bar{S}=\left(S_{1}+S_{0}\right) / 2$.

\section{Appendix D. The diffraction integral for the period-tripling bifurcation}

In this section we evaluate the diffraction integral which appears in the uniform approximation for the period-tripling bifurcation. We consider first the case $z>0$ :

$$
\begin{aligned}
\int_{0}^{\infty} \mathrm{d} I J_{0}\left(I^{3 / 2}\right) \mathrm{e}^{\mathrm{i} z I}=\lim _{\varepsilon \rightarrow 0} \sum_{n=0}^{\infty} \frac{(\mathrm{i} z)^{n}}{n !} \int_{0}^{\infty} \mathrm{d} I I^{n} J_{0}\left(I^{3 / 2}\right) \mathrm{e}^{-\varepsilon I^{3 / 2}} \\
=\lim _{\varepsilon \rightarrow 0} \sum_{n=0}^{\infty} \frac{(\mathrm{i} z)^{n}}{n !} \frac{2}{3}\left(1+\varepsilon^{2}\right)^{-(n+1) / 3} \Gamma\left(\frac{2 n}{3}+\frac{2}{3}\right) P_{(2 n-1) / 3}\left(\frac{\varepsilon}{\sqrt{1+\varepsilon^{2}}}\right) \\
=\frac{2}{3 \pi} \sum_{n=0}^{\infty} \frac{(\mathrm{i} z)^{n}}{n !} 2^{(2 n-1) / 3} \Gamma^{2}\left(\frac{n}{3}+\frac{1}{3}\right) \sin \left(\frac{\pi n}{3}+\frac{\pi}{3}\right) \\
=\frac{2}{3} \sum_{n=0}^{\infty}\left(\frac{4 \mathrm{i} z^{3}}{27}\right)^{n} 2^{-1 / 3} \frac{\Gamma\left(n+\frac{1}{3}\right)}{n ! \Gamma\left(n+\frac{2}{3}\right)}+\frac{2 \mathrm{i} z}{3} \sum_{n=0}^{\infty}\left(\frac{4 \mathrm{i} z^{3}}{27}\right)^{n} 2^{1 / 3} \frac{\Gamma\left(n+\frac{2}{3}\right)}{3 n ! \Gamma\left(n+\frac{4}{3}\right)} \\
=\frac{2^{2 / 3} \Gamma\left(\frac{1}{3}\right)}{3 \Gamma\left(\frac{2}{3}\right)}{ }_{1} F_{1}\left(\frac{1}{3} ; \frac{2}{3} ; \frac{4 \mathrm{i} z^{3}}{27}\right)+\mathrm{i} z \frac{2^{4 / 3} \Gamma\left(\frac{2}{3}\right)}{9 \Gamma\left(\frac{4}{3}\right)}{ }_{1} F_{1}\left(\frac{2}{3} ; \frac{4}{3} ; \frac{4 i z^{3}}{27}\right)
\end{aligned}
$$




$$
=\frac{2}{3} \sqrt{\frac{\pi z}{3}} \exp \left(\frac{2 \mathrm{i} z^{3}}{27}\right)\left[J_{-1 / 6}\left(\frac{2 z^{3}}{27}\right)+\mathrm{i} J_{1 / 6}\left(\frac{2 z^{3}}{27}\right)\right] .
$$

The parameter $\varepsilon$ has been introduced in (71) in order to make the integrals absolutely convergent. The integrals leading to (72) after a substitution $x=I^{3 / 2}$ can be found in [31]. From (72) to (73) the limit $\varepsilon \rightarrow 0$ has been performed and the duplication formula of the gamma function has been used. From (73) to (74) the sum has been split into three parts (by taking every third term, respectively) where the third part vanished. Furthermore, the recurrence formula and the triplication formula of the gamma function have been used. From (74) to (75) the definition of Kummer's function ${ }_{1} F_{1}$ has been used, and from (75) to (76) the formula $\exp (\mathrm{i} z) J_{v}(z)=(z / 2){ }_{1} F_{1}\left(v+\frac{1}{2} ; 2 v+1 ; 2 \mathrm{i} z\right) / \Gamma(v+1)$ was used [32].

The corresponding expression for negative values of $z$ follows from the evenness and oddness of the real and imaginary part of the integral, respectively. We obtain

$$
\int_{0}^{\infty} \mathrm{d} I J_{0}\left(a I^{3 / 2}\right) \mathrm{e}^{-\mathrm{i} z I}=\frac{2}{3} \sqrt{\frac{\pi|z|}{3 a^{2}}} \exp \left\{-\mathrm{i} \frac{2 z^{3}}{27 a^{2}}\right\}\left[J_{-1 / 6}\left(\frac{2|z|^{3}}{27 a^{2}}\right)-\mathrm{i} \operatorname{sign}(z) J_{1 / 6}\left(\frac{2|z|^{3}}{27 a^{2}}\right)\right]
$$

and from the derivative of this integral with respect to $z$ follows

$$
\begin{aligned}
\int_{0}^{\infty} \mathrm{d} I I J_{0}\left(a I^{3 / 2}\right) \mathrm{e}^{-\mathrm{i} z I}=\frac{4}{27} \sqrt{\frac{\pi|z|^{5}}{3 a^{6}}} \exp \left\{-\mathrm{i} \frac{2 z^{3}}{27 a^{2}}\right\} \\
\times\left[J_{-1 / 6}\left(\frac{2|z|^{3}}{27 a^{2}}\right)-\mathrm{i} \operatorname{sign}(z) J_{1 / 6}\left(\frac{2|z|^{3}}{27 a^{2}}\right)\right. \\
\left.+J_{-5 / 6}\left(\frac{2|z|^{3}}{27 a^{2}}\right)-\mathrm{i} \operatorname{sign}(z) J_{5 / 6}\left(\frac{2|z|^{3}}{27 a^{2}}\right)\right] .
\end{aligned}
$$

\section{References}

[1] Gutzwiller M C 1971 Periodic orbits and classical quantization conditions J. Math. Phys. 12 343-58

[2] Balian R and Bloch C 1972 Distribution of eigenfrequencies for the wave equation in a finite domain: III. Eigenfrequency density oscillations Ann. Phys. 69 76-160

[3] Balian R and Bloch C 1974 Solution of the Schrödinger equation in terms of classical paths Ann. Phys. 85 514-45

[4] Berry M V and Tabor M 1976 Closed orbits and the regular bound spectrum Proc. R. Soc. A 349 101-23

[5] Berry M V and Tabor M 1977 Calculating the bound spectrum by path summation in action-angle variables J. Phys. A: Math. Gen. 10 371-9

[6] Gutzwiller M C 1990 Chaos in Classical and Quantum Mechanics (New York: Springer)

[7] Creagh S C and Littlejohn R G 1991 Semiclassical trace formulas in the presence of continuous symmetries Phys. Rev. A 44 836-50

[8] Creagh S C and Littlejohn R G 1992 Semiclassical trace formulae for systems with non-Abelian symmetry J. Phys. A: Math. Gen. 25 1643-69

[9] de Almeida A M O and Hannay J H 1987 Resonant periodic orbits and the semiclassical energy spectrum $J$. Phys. A: Math. Gen. 20 5873-83

[10] Meyer K R 1970 Generic bifurcations of periodic points Trans. Am. Math. Soc. 149 95-107

[11] Bruno A D 1970 Instability in a Hamiltonian system and the distribution of asteroids Math. USSR Sbornik 12 271-312

[12] Bruno A D Research on the restricted three body problem. I: Periodic solutions of a Hamiltonian system Preprint 18 (Moscow: Inst. Prikl. Mat. Akad. Nauk SSSR.) 44pp (in Russian)

[13] Sieber M 1996 Uniform approximation for bifurcations of periodic orbits with high repetition numbers $J$. Phys. A: Math. Gen. 29 4715-32

[14] Sieber M 1997 Semiclassical transition from an elliptical to an oval billiard J. Phys. A: Math. Gen. 30 4563-96 
[15] Miller W H 1974 Classical-limit quantum mechanics and the theory of molecular collisions Adv. Chem. Phys. 25 69-177

[16] Littlejohn R G 1990 Semiclassical structure of trace formulas J. Math. Phys. 31 2952-77

[17] Sadovskií D A and Delos J B 1996 Bifurcation of the periodic orbits of Hamiltonian systems: an analysis using normal form theory Phys. Rev. E 54 2033-70

[18] Bogomolny E, Bohigas O and Leboeuf P 1992 Distribution of roots of random polynomials Phys. Rev. Lett. $682726-9$

[19] Haake F, Kuś M and Scharf R 1987 Classical and quantum chaos for a kicked top Z. Phys. B 65 381-95

[20] Kuś M, Haake F and Eckhardt B 1993 Quantum effects of periodic orbits for the kicked top Z. Phys. B 92 221-33

[21] Braun P A, Gerwinski P, Haake F and Schomerus H 1996 Semiclassics of rotation and torsion Z. Phys. B $100115-27$

[22] Kuś M, Haake F and Delande D 1993 Prebifurcation periodic ghost orbits in semiclassical quantization Phys. Rev. Lett. 71 2167-71

[23] Arnol'd V I 1973 Remarks on the stationary phase method and Coxeter numbers Russ. Math. Surv. 28 19-48

[24] Sadovskií D A, Shaw J A and Delos J B 1995 Organization of sequences of bifurcations of periodic orbits Phys. Rev. Lett. 75 2120-3

[25] Schomerus H 1997 Semiclassical interference of bifurcations Preprint chao-dyn/9703003

[26] Rimmer R J 1982 Generic bifurcations for involutory area preserving maps Memoirs of the AMS no 272 (Providence, RI: American Mathematical Society)

[27] Aguiar M A M D, Malta C P, Baranger M and Davies K T R 1987 Bifurcations of periodic trajectories in non-integrable Hamiltonian systems with two degrees of freedom: numerical and analytical results Ann. Phys. 180 167-205

[28] Poston T and Stewart I N 1978 Catastrophe Theory and its Applications (London: Pitman)

[29] Mouchet A 1996 Quelques applications des méthodes semiclassiques en chaos quantique PhD Thesis Université Paris 6

[30] Abramowitz M and Stegun I A (ed) 1965 Handbook of Mathematical Functions (New York: Dover)

[31] Prudnikov A P, Brychkov Y A and Marichev O I 1988 Integrals and Series, Volume 2: Special Functions (New York: Gordon and Breach) 2nd printing with corrections

[32] Magnus W, Oberhettinger F and Soni R P 1966 Formulas and Theorems for the Special Functions of Mathematical Physics (Berlin: Springer) 